\title{
EXACT ADAPTIVE POINTWISE ESTIMATION ON SOBOLEV CLASSES OF DENSITIES
}

\author{
Cristina ButuceA ${ }^{1}$
}

\begin{abstract}
The subject of this paper is to estimate adaptively the common probability density of $n$ independent, identically distributed random variables. The estimation is done at a fixed point $x_{0} \in \mathbb{R}$, over the density functions that belong to the Sobolev class $W_{n}(\beta, L)$. We consider the adaptive problem setup, where the regularity parameter $\beta$ is unknown and varies in a given set $B_{n}$. A sharp adaptive estimator is obtained, and the explicit asymptotical constant, associated to its rate of convergence is found.
\end{abstract}

Mathematics Subject Classification. 62N01, 62N02, 62G20.

Received November 15, 2000. Revised April 9, 2001.

\section{INTRODUCTION}

Consider $n$ independent, identically distributed random variables $X_{1}, \ldots, X_{n}$, having common unknown probability density $f: \mathbb{R} \rightarrow[0, \infty)$. We assume that $f$ belongs to a Sobolev class of densities.

For any $L>0$ and $\beta$ positive integer, we define the Sobolev class of densities $W(\beta, L)$, as the set of functions

$$
W(\beta, L)=\left\{f: \mathbb{R} \rightarrow[0, \infty): \int_{\mathbb{R}} f=1, \int_{\mathbb{R}}\left(f^{(\beta)}(x)\right)^{2} \mathrm{~d} x \leq L^{2}\right\}
$$

where $f^{(\beta)}$ will denote from now on the generalized derivative of order $\beta$ of $f$. We may define for an absolutely integrable function $f: \mathbb{R} \rightarrow \mathbb{R}$ its Fourier transform $\mathcal{F}(f)(x)=\int_{\mathbb{R}} f(y) \mathrm{e}^{-i x y} \mathrm{~d} y$, for any $x$ in $\mathbb{R}$. We adopt now a more general definition of the Sobolev class, allowing non-integer values of $\beta>1 / 2$

$$
W(\beta, L)=\left\{f: \mathbb{R} \rightarrow[0, \infty): \int_{\mathbb{R}} f=1, \int_{\mathbb{R}}|\mathcal{F}(f)(x)|^{2}|x|^{2 \beta} \mathrm{d} x \leq 2 \pi L^{2}\right\}
$$

Let $f_{n}$ be an estimator of $f$ based on the sample $X_{1}, \ldots, X_{n}$ and $x_{0}$ a fixed point. The performance of the estimator $f_{n}$ at the point $x_{0}$ is measured by the maximal risk

$$
R_{n, \beta}^{*}\left(f_{n}, \varphi_{n, \beta}\right)=\sup _{f \in W(\beta, L)} E_{f}\left[\varphi_{n, \beta}^{-q}\left|f_{n}\left(x_{0}\right)-f\left(x_{0}\right)\right|^{q}\right]
$$

\footnotetext{
Keywords and phrases: Density estimation, exact asymptotics, pointwise risk, sharp adaptive estimator.

${ }^{1}$ Université Paris 10, Modal'X, bâtiment G, 200 avenue de la République, 92001 Nanterre, France; e-mail: cbutucea@u-paris10.fr and Université Paris 6, Laboratoire Probabilités et Modèles Aléatoires, 6 rue Clisson, 75013 Paris, France; e-mail: butucea@ccr.jussieu.fr
} 
where $E_{f}(\cdot)$ is the expectation with respect to the distribution $P_{f}$ of $X_{1}, \ldots, X_{n}$, when the underlying probability density is $f, \varphi_{n, \beta}$ is a given sequence of positive numbers and $q>0$.

The sequence $\varphi_{n, \beta}$ such that the maximal risk related to (1.1) remains positive for all estimation procedures $f_{n}$, asymptotically, and finite for some explicit estimator, asymptotically, is called the optimal rate of convergence for the class $W(\beta, L)$. Using the argument of optimal recovery as in Donoho and Low [10], it is easy to find that the optimal pointwise rate of convergence over the Sobolev class $W(\beta, L)$ is $\varphi_{n, \beta}=(1 / n)^{\frac{\beta-1 / 2}{2 \beta}}$. This rate and the estimator attaining this rate depend on the regularity $\beta$ of the unknown density $f$. Thus, it is difficult to implement such an estimator in practice. Our goal is to suggest an adaptive estimator $f_{n}\left(x_{0}\right)$ of $f\left(x_{0}\right), x_{0} \in \mathbb{R}$, i.e. an estimator independent of the regularity $\beta$ of $f$, which is optimal in an exact asymptotic sense.

To define the notion of adaptive optimality we follow the minimax framework applied to the problem of adaptivity by Lepskii [23]. He considered the Gaussian white noise model, rather than density estimation. In this context, he introduced the notions of adaptive rate of convergence and rate adaptive estimator.

Adaptive rates of convergence on different functional classes for the Gaussian white noise model were obtained by Donoho et al. [8] (who give a detailed overview of the results in adaptive estimation), by Lepski et al. [27], Goldenshluger and Nemirovski [13], Juditsky [19]. Most of these results relate to Besov classes of functions. The latter three papers use the Lepski type of adaptation.

For the same framework of the Gaussian white noise model, exact adaptive results are available for several cases. Exact adaptivity means that not only the rate but the best asymptotic constant associated to it is attained by the proposed adaptive estimator. The first result of this kind in the estimation in $\mathbb{L}_{2}$ norm on Sobolev periodic classes belongs to Efromovich and Pinsker [12]. For further developments see Golubev [14,15], Golubev and Nussbaum [17]. Then Lepskii [23], Lepski and Spokoiny [28] obtained exact adaptive results in $\mathbb{L}_{\infty}$ and at a fixed point, respectively, on the Hölder classes with $0<\beta \leq 2$ (see also Lepskii [24] and [25]). Tsybakov [30] proved exact adaptive results for the Gaussian white noise model both in $\mathbb{L}_{\infty}$ and at a fixed point, on the Sobolev classes. Lepski and Levit [26] gave exact adaptive results in pointwise estimation over a large scale of infinitely differentiable functions.

Similar results on the adaptive rates of convergence exist in density estimation. We refer here to Donoho et al. [9], Kerkyacharian et al. [21] and Juditsky [19] for the general setting of Besov classes and $\mathbb{L}_{p}$ norm, with $p<\infty$. They applied the wavelet shrinkage in order to construct the adaptive estimator. Barron et al. [1], Birgé and Massart [3] propose different adaptive density estimators constructed by the methods of penalization and prove their adaptivity in the $\mathbb{L}_{2}$ norm. Devroye and Lugosi [7] obtained similar results for the $\mathbb{L}_{1}$ norm using an original adaptation procedure.

The results on exact adaptive estimation of the density of i.i.d. random variables, under the quadratic $\mathbb{L}_{2}$ risk, are due to Efromovich [11] and Golubev [16]. Efromovich [11] considered estimation over Sobolev and more general ellipsoids of periodic densities on $[0,1]$, Golubev [16] estimated a density in Sobolev classes over the real line.

In this paper, we consider the problem of exact adaptive density estimation at a fixed point on the Sobolev classes. Note that, similarly to the results of Lepskii [23], Brown and Low [4] concerning the pointwise estimation in Gaussian white noise model, the adaptive estimation with the optimal rate $\varphi_{n, \beta}=(1 / n)^{\frac{\beta-1 / 2}{2 \beta}}$ is not possible and we have to normalize the risk by the adaptive rate of convergence, which is logarithmically worse than the optimal rate. In fact, the adaptive rate of convergence on a slightly modified Sobolev class $W_{n}(\beta, L)$, described below, is of the order $(\log n / n)^{\frac{\beta-1 / 2}{2 \beta}}$ (see Butucea [5]). The main result of this paper is to find the constant $c\left(\beta, L, q, f\left(x_{0}\right)\right)$ multiplying this rate of convergence which allows to attain the exact asymptotics of the minimax adaptive risk. We also construct explicitly the adaptive estimator that attains this exact asymptotics. This extends a pointwise adaptive result of Tsybakov [30] to the problem of density estimation. 


\section{Results}

Our results concern the exact adaptive estimation, at a fixed point $x_{0} \in \mathbb{R}$, over the Sobolev class of densities, with regularity $\beta \in B_{n}$, for some set $B_{n}$. We introduce the following class of Sobolev densities

$$
W_{n}(\beta, L)=\left\{f \in W(\beta, L): f\left(x_{0}\right) \geq \rho_{n}\right\},
$$

where $\rho_{n}$ is a sequence of positive real numbers that satisfies

$$
\lim _{n \rightarrow \infty} \rho_{n}=0 \text { and } \liminf _{n \rightarrow \infty}\left(\rho_{n} \log n\right)>0
$$

This truncation prevents us from the possible case of a density $f$ that varies with $n$ such that $f\left(x_{0}\right) \rightarrow 0$ too fast as $n \rightarrow \infty$.

An alternative possibility is to consider that our density belongs to a local Sobolev class, where the smoothness on a neighborhood around the estimation point is quantified. In this context only the adaptive rate of convergence is maintained by the estimation approach described here and not the exact constant normalization.

Consider now the following maximal risk over $W_{n}(\beta, L)$ at fixed $x_{0}$, for $q>0$

$$
R_{n, \beta}\left(f_{n}, \psi_{n, \beta}\right)=\sup _{f \in W_{n}(\beta, L)} E_{f}\left[\psi_{n, \beta}^{-q}\left|f_{n}\left(x_{0}\right)-f\left(x_{0}\right)\right|^{q}\right]
$$

We assume that the set $B_{n}$ of regularities is a discrete set, $B_{n}=\left\{\beta_{1}, \ldots, \beta_{N_{n}}\right\}$, where $1 / 2<\beta_{1}<\ldots<\beta_{N_{n}}$ $<+\infty$ are positive integers. Moreover, we suppose that $\beta_{1}>1 / 2$ is fixed, while $\lim _{n \rightarrow \infty} \beta_{N_{n}}=+\infty$ and $\left\{N_{n}\right\}_{n \geq 1}$ is a nondecreasing sequence of positive integers. We define $\Delta_{n}=\max _{i=1 \ldots N_{n}-1}\left|\beta_{i+1}-\beta_{i}\right|$ and assume that it satisfies

$$
\limsup _{n \rightarrow \infty} \Delta_{n}<+\infty
$$

together with

$$
\lim _{n \rightarrow \infty} \frac{\Delta_{n} \log n}{\beta_{N_{n}}^{2} \log \log n}=\infty .
$$

Considering a discrete set $B_{n}$ of regularities is not only a technical matter. Similarly to Lepski's result, we may as well consider a bounded set of regularities $[a, b] \subseteq(1 / 2, \infty)$. This changes nothing to the adaptive rate of convergence, but a factor $\frac{1}{2 \beta}-\frac{1}{2 b}$ should appear in the constant (where $\beta$ is the true underlying regularity). We refer to Klemelä and Tsybakov [22] for such type of sharp normalizations. A continuous set of values $[a, b]$ would reduce to the same techniques by considering a grid of values on this interval which is growing finer with $n$. From a practical point of view, the estimation method described later works on discretized sets of regularities. Our approach considers a larger union of classes, where $\beta_{N_{n}}$ goes to infinity, without loss in the rate.

In particular, conditions (2.3) and (2.4) entail

$$
\begin{array}{r}
\frac{\log n}{\beta_{N_{n}}} \underset{n \rightarrow \infty}{\rightarrow}+\infty \\
\left(\frac{\beta_{N_{n}}}{\log n}\right)^{1 / 2} \log \beta_{N_{n}} \underset{n \rightarrow \infty}{\rightarrow} 0 \\
\left(\frac{\beta_{N_{n}}}{\log n}\right)^{1 / 2} \log \frac{1}{\Delta_{n}} \underset{n \rightarrow \infty}{\rightarrow} 0 .
\end{array}
$$

This is proved in the beginning of Section 3. 
The following definition is a modification for the density problem of the adaptive optimality introduced by Lepskii [23] (see also Tsybakov [30]).

Definition 2.1. The sequence $\psi_{n, \beta}$ is an adaptive rate of convergence over the set $B_{n}$, if:

1. there exists an estimator $f_{n}^{*}$, independent of $\beta$ over $B_{n}$, which is called rate adaptive estimator, such that

$$
\limsup _{n \rightarrow \infty} \sup _{\beta \in B_{n}} R_{n, \beta}\left(f_{n}^{*}, \psi_{n, \beta}\right)<\infty ;
$$

2. if there exists another sequence of positive real numbers $\rho_{n, \beta}$ and an estimator $f_{n}^{* *}$ such that

$$
\limsup _{n \rightarrow \infty} \sup _{\beta \in B_{n}} R_{n, \beta}\left(f_{n}^{* *}, \rho_{n, \beta}\right)<\infty
$$

and, at some $\beta^{\prime}$ in $B_{n}, \frac{\rho_{n, \beta^{\prime}}}{\psi_{n, \beta^{\prime}}} \underset{n \rightarrow \infty}{\rightarrow} 0$, then there is another $\beta^{\prime \prime}$ in $B_{n}$ such that $\frac{\rho_{n, \beta^{\prime}}}{\psi_{n, \beta^{\prime}}} \cdot \frac{\rho_{n, \beta^{\prime \prime}}}{\psi_{n, \beta^{\prime \prime}}} \underset{n \rightarrow \infty}{\rightarrow}+\infty$.

Note that condition (2.8) introduces a wide class of rates. We choose between those rates by a criterion of uniformity over the set $B_{n}$, expressed in the second part of Definition 2.1. If some other rate satisfies a condition similar to $(2.8)$ and if this rate is faster at some point $\beta^{\prime}$ then the loss at some other point $\beta^{\prime \prime}$ has to be infinitely greater for large sample sizes $n$.

Let us denote $B_{-}=B_{n} \backslash\left\{\beta_{N_{n}}\right\}$ and

$$
\psi_{n, \beta}=\left\{\begin{array}{c}
c\left(\frac{\log n}{n}\right)^{\frac{\beta-1 / 2}{2 \beta}}, \text { for } \beta \in B_{-} \\
\left(\frac{1}{n}\right)^{\frac{\beta-1 / 2}{2 \beta}}, \text { for } \beta=\beta_{N_{n}},
\end{array}\right.
$$

where the constant $c$ is function of $\beta, L, q$ and $f\left(x_{0}\right), c=c\left(\beta, L, q, f\left(x_{0}\right)\right)>0$ and satisfies

$$
0<\liminf _{\beta \rightarrow \infty} c\left(\beta, L, q, f\left(x_{0}\right)\right) \sqrt{\beta} \leq \limsup _{\beta \rightarrow \infty} c\left(\beta, L, q, f\left(x_{0}\right)\right) \sqrt{\beta}<\infty .
$$

\subsection{Exact adaptive estimation procedure}

The estimation procedure contains three steps. First, we consider a preliminary estimator $\widehat{f}_{n}\left(x_{0}\right)$ of $f\left(x_{0}\right)$, as the following kernel estimator

$$
\widehat{f}_{n}\left(x_{0}\right)=\frac{1}{n h_{n}} \sum_{i=1}^{n} K\left(\frac{X_{i}-x}{h_{n}}\right)
$$

where $K$ is a bounded, positive kernel, such that $\int|u||K(u)| \mathrm{d} u<\infty$, and the bandwidth is $h_{n}>0$. Let us mention that the regularity $\beta$ of $f$ being greater than $1 / 2$ the density is regular enough in order to be pointwise evaluable.

The bandwidth satisfies $\lim _{n \rightarrow \infty} h_{n}=0$ and $\lim _{n \rightarrow \infty} n h_{n}=\infty$, which guarantee that $\widehat{f}_{n}\left(x_{0}\right)$ is a consistent estimator. This preliminary estimator does not need to be particularly well performing, but for technical reasons in Lemma 3.2 it must not be too slow. Let us consider a preliminary bandwidth such that

$$
h_{n}=O\left(n^{-\alpha_{0}}\right)
$$

for some fixed $0<\alpha_{0}<1 / 2$. We truncate this estimator at $\rho_{n}$ that tends to 0 when $n \rightarrow \infty$ such that (2.1) holds and get $\widehat{\rho}_{n}\left(x_{0}\right)=\max \left\{\widehat{f}_{n}\left(x_{0}\right), \rho_{n}\right\}$. 
The second step consists in defining a family of kernel estimators, whose bandwidths contain the preliminary estimator as follows

$$
\begin{aligned}
\widehat{h}_{n, \beta} & =k_{\beta}\left(\frac{\widehat{\rho}_{n}\left(x_{0}\right) \log n}{n}\right)^{\frac{1}{2 \beta}}, \text { where } \beta \in B_{-} \text {and } k_{\beta}=\left(\frac{q}{2 \beta(2 \beta-1) L^{2}}\right)^{\frac{1}{2 \beta}} \\
\widehat{h}_{n, \beta_{N_{n}}} & =\left(\frac{1}{n}\right)^{\frac{1}{2 \beta_{N_{n}}}} \text {, if } \beta=\beta_{N_{n}} .
\end{aligned}
$$

For $\beta>1 / 2$, define a kernel $K_{\beta}$ by the expressions

$$
K_{\beta}(x)=\frac{1}{2 \pi} \int_{\mathbb{R}} \frac{\exp (i x u)}{1+|u|^{2 \beta}} \mathrm{d} u=\frac{1}{\pi} \int_{0}^{\infty} \frac{\cos (x u)}{1+|u|^{2 \beta}} \mathrm{d} u .
$$

Define the kernel estimator depending on $\beta$

$$
\widehat{f}_{n, \beta}\left(x_{0}\right)=\frac{1}{n \widehat{h}_{n, \beta}} \sum_{i=1}^{n} K_{\beta}\left(\frac{X_{i}-x_{0}}{\widehat{h}_{n, \beta}}\right) .
$$

At the third step, we use a version of Lepski's method to approximate $\beta$ by an estimator $\widehat{\beta}$ and to substitute it in the expression of the kernel estimator $\widehat{f}_{n, \beta}\left(x_{0}\right)$. For any $\beta>1 / 2$, we define

$$
b_{\beta}^{2}=\frac{1}{2 \pi} \int_{\mathbb{R}} \frac{|u|^{2 \beta}}{\left(1+|u|^{2 \beta}\right)^{2}} \mathrm{~d} u, \nu_{\beta}^{2}=\frac{1}{2 \pi} \int_{\mathbb{R}} \frac{1}{\left(1+|u|^{2 \beta}\right)^{2}} \mathrm{~d} u .
$$

By simple calculation, we can prove that $\nu_{\beta}^{2}=(2 \beta-1) b_{\beta}^{2}$. We consider the sequence

$$
\widehat{\eta}_{n, \beta}=\nu_{\beta} \sqrt{\frac{q}{2 \beta k_{\beta}}}\left(\frac{\widehat{\rho}_{n}\left(x_{0}\right) \log n}{n}\right)^{\frac{\beta-1 / 2}{2 \beta}}
$$

and the estimator of $\beta$ defined as

$$
\hat{\beta}=\max \left\{\beta \in B_{n}:\left|\widehat{f}_{n, \gamma}\left(x_{0}\right)-\widehat{f}_{n, \beta}\left(x_{0}\right)\right| \leq \widehat{\eta}_{n, \gamma}, \forall \gamma \in B_{n}, \gamma \leq \beta\right\} .
$$

Finally, we replace $\beta$ by $\hat{\beta}$ in the kernel estimator $\widehat{f}_{n, \beta}\left(x_{0}\right)$ in order to get the estimator

$$
f_{n}^{*}\left(x_{0}\right)=\widehat{f}_{n, \hat{\beta}}\left(x_{0}\right) .
$$

This estimator will be shown to be exact adaptive i.e. to attain the adaptive rate of convergence in our setup, up to a constant, explicitly given.

The key point in this construction is Lepski's algorithm in the third step for the evaluation of the smoothness $\beta$ of the underlying density, at each point $x_{0}$. The same method was employed by Tsybakov [30] in the Gaussian white noise model but for orthogonal series estimators of the signal function. We introduce here kernel estimators which are more suitable for density estimation and are largely used in practice. Moreover, we provide optimal kernel (in the sense of exact adaptivity) and bandwidth expression of the adaptive estimator.

For a simulation study of the behavior of this estimator we refer to Butucea [6]. We have implemented the described adaptive estimator and tested it over ten different densities. We have considered a set $B=\{1, \ldots, 6\}$ and $L=10$. As a preliminary estimator we used a Gaussian kernel estimator, with a rather large bandwidth. 
We came to the conclusion that the adaptive estimator is particularly robust with respect to this preliminary estimator. Also, the choice of $L$ is not of crucial importance. Choosing $L$ adaptively reduces to taking a finer grid on $\beta$. The local sharp adaptive estimator behaves uniformly well for many different densities in the described class. Also, this study cleared out the behaviour of this procedure for more general analytic functions, as the classical Gaussian density. This function belongs to each Sobolev class $W(\beta, L)$ (where $L$ gets larger with $\beta$ ) and $\hat{\beta}$ chooses $\beta_{N_{n}}$ (for large enough $L$ ). The adaptive estimator achieves the rate $n^{-\left(\beta_{N_{n}}-1 / 2\right) / \beta_{N_{n}}}$ which is close to $n^{-1 / 2}$ for large $\beta_{N_{n}}$.

Finally, a major difference with respect to the nonparametric regression or Gaussian white noise model, as considered in Lepski and Spokoiny [28], Tsybakov [30], is the fact that the density model is heteroscedastic. This means more precisely that the variance of the kernel estimator is proportional to $f\left(x_{0}\right)$, the value of the unknown density at the estimation point. In consequence, this value appears into our exact normalization and optimal estimators and hence the use of a preliminary estimator of $f\left(x_{0}\right)$, which has to be free of unknown values $f\left(x_{0}\right)$ and $\beta$.

\subsection{Statement of results}

Let $c=c\left(\beta, L, q, f\left(x_{0}\right)\right)$ be a positive constant defined by

$$
c\left(\beta, L, q, f\left(x_{0}\right)\right)=b_{\beta} L^{\frac{1}{2 \beta}}(2 \beta)^{\frac{\beta+1 / 2}{2 \beta}}\left(\frac{q f\left(x_{0}\right)}{2 \beta-1}\right)^{\frac{\beta-1 / 2}{2 \beta}} .
$$

Theorem 2.2. The estimator $f_{n}^{*}\left(x_{0}\right)$ defined by (2.13) is rate adaptive estimator and the adaptive rate $\psi_{n, \beta}$ associated to the constant $c\left(\beta, L, q, f\left(x_{0}\right)\right)$ in (2.14) is such that

$$
\limsup _{n \rightarrow \infty} \sup _{\beta \in B_{-}} R_{n, \beta}\left(f_{n}^{*}, \psi_{n, \beta}\right) \leq 1
$$

and

$$
\liminf _{n \rightarrow \infty} \inf _{f_{n}} \sup _{\beta \in B_{-}} R_{n, \beta}\left(f_{n}, \psi_{n, \beta}\right) \geq 1
$$

Moreover,

$$
\limsup _{n \rightarrow \infty} R_{n, \beta_{N_{n}}}\left(f_{n}^{*}, \psi_{n, \beta_{N_{n}}}\right)<\infty
$$

This exact constant is obviously similar to the case of Gaussian white noise model in Tsybakov [30]. Nevertheless, as we stressed in our introduction very few results of this type are known in the density estimation model.

The pointwise estimation allows more flexibility than the global adaptation and, in particular, the bandwidth and the kernel can be adjusted locally. Moreover, the adaptive setup in the pointwise estimation is more interesting because the rates are significantly different (slower by a $\log n$ factor) than the nonadaptive rates, except at the last point of the set $B_{N_{n}}$. This is usually not happening in global $\mathbb{L}_{p}$ estimation.

Our theorem states that $\psi_{n, \beta}$ and $f_{n}^{*}$ satisfy the exact adaptive problem on $B_{-}$:

$$
\lim _{n \rightarrow \infty} \inf _{f_{n}} \sup _{\beta \in B_{-}} R_{n, \beta}\left(f_{n}, \psi_{n, \beta}\right)=\lim _{n \rightarrow \infty} \sup _{\beta \in B_{-}} R_{n, \beta}\left(f_{n}^{*}, \psi_{n, \beta}\right)=1
$$

split in two inequalities (2.15) - also called upper bound and (2.16) - known as the lower bound. The same procedure attains the optimal rate of convergence at the last point of the set, $\beta_{N_{n}}$.

The proof of the upper bounds (2.15) and (2.17) is given in Section 5. The different tools and techniques that are used here are described in Sections 3 and 4. In Section 3, we study the preliminary estimator and give 
exact bounds for the bias and the variance of the optimal kernel estimator $f_{n, \beta}$. Section 4 contains the main tools in our proof, stated as theorems.

On one hand, in order to bound uniform risks of the adaptive estimator both exponential and uniform exponential inequalities are needed (Bernstein's and, respectively, van de Geer's inequalities). An original technique is used in Theorem 4.6, where we split the integration domain of the risk and treat each case differently. A global entropy reasoning or chaining would not provide us the right constants. On the other hand, it is necessary to study the estimator of $\beta$ and to quantify exactly the probability that $\widehat{\beta}$ is strictly less than the true $\beta$. We see that this event happens with exponentially small probability.

Section 6 gives a constructive proof of the lower bounds. Namely, the subexperiments that are difficult enough and lead to the exact lower bounds are given.

\section{Auxiliary Results}

From now on $o(1)$ denotes any sequence that tends to 0 when $n \rightarrow \infty, O(1)$ and $d_{i}, i=0,1,2, \ldots$ are positive constants, depending eventually on fixed, positive $\beta_{1}, q$ and $L$, while $c_{i}, i=1,2, \ldots$ are absolute positive constants.

Proof of (2.5) to (2.7). We apply (2.3) and (2.4) and we pass to limits:

$$
\frac{\beta_{N_{n}}}{\log n}=\frac{\beta_{N_{n}}^{2} \log \log n}{\Delta_{n} \log n} \frac{\Delta_{n}}{\beta_{N_{n}} \log \log n} \leq o(1) \limsup _{n \rightarrow \infty} \Delta_{n}=o(1)
$$

Next, for $n$ large enough, $\beta_{N_{n}} \log ^{2} \beta_{N_{n}} \leq \beta_{N_{n}}^{2} \log \beta_{N_{n}} \leq \beta_{N_{n}}^{2} \log \log n$ and then

$$
\left(\frac{\beta_{N_{n}}}{\log n}\right)^{1 / 2} \log \beta_{N_{n}} \leq\left(\frac{\beta_{N_{n}}^{2} \log \log n}{\log n}\right)^{1 / 2} \leq\left(\Delta_{n} \frac{\beta_{N_{n}}^{2} \log \log n}{\Delta_{n} \log n}\right)^{1 / 2} \underset{n \rightarrow \infty}{\rightarrow} 0 .
$$

Finally, we remark that for $n$ large enough, $\frac{1}{\Delta_{n}} \leq \log n$ and then $\log \frac{1}{\Delta_{n}} \leq \log \log n$. We also have $\log \frac{1}{\Delta_{n}} \leq \frac{1}{\Delta_{n}}$ and we write $\log \frac{1}{\Delta_{n}} \leq\left(\frac{\log \log n}{\Delta_{n}}\right)^{1 / 2}$. Then

$$
\left(\frac{\beta_{N_{n}}}{\log n}\right)^{1 / 2} \log \frac{1}{\Delta_{n}} \leq\left(\frac{1}{\beta_{N_{n}}} \frac{\beta_{N_{n}}^{2} \log \log n}{\Delta_{n} \log n}\right)^{1 / 2} \underset{n \rightarrow \infty}{\rightarrow} 0 .
$$

Let us remark that the kernel $K_{\beta}$ in (2.12) has the Fourier transform

$$
\mathcal{F}\left(K_{\beta}\right)(u)=\frac{1}{1+|u|^{2 \beta}}
$$

and by Plancherel formula $\left\|K_{\beta}\right\|_{2}^{2}=\frac{1}{2 \pi}\left\|\mathcal{F}\left(K_{\beta}\right)\right\|_{2}^{2}=\nu_{\beta}^{2}$.

Lemma 3.1. There exist positive constants $K_{\max }, k_{\max }, k_{\min }, \nu_{\max }, \nu_{\min }$ and $v_{\max }$ depending only on fixed $\beta_{1}$, $q$ and $L$ such that:

1. $\left\|K_{\beta}\right\|_{\infty} \leq K_{\max }, k_{\min } \leq k_{\beta} \leq k_{\max }$ and $\nu_{\min } \leq \nu_{\beta} \leq \nu_{\max }$, for any $\beta$ in $B_{n}$;

2. $\max _{\beta \in B} \sup _{f \in W_{n}(\beta, L)}\|f\|_{\infty} \leq v_{\max }$. 
Proof. 1. We shall prove only the first statement, the rest being an easy consequence of the definition of $k_{\beta}$ and $\nu_{\beta}$. We have for $2 \beta_{1}>1$ :

$$
\left\|K_{\beta}\right\|_{\infty} \leq \frac{1}{2 \pi}\left(1+\int_{|u|>1} \frac{\mathrm{d} u}{1+|u|^{2 \beta_{1}}}\right)=K_{\max }\left(\beta_{1}\right)<+\infty
$$

2. For $\beta>1 / 2, f$ in $W_{n}(\beta, L)$ is a continuous function and

$$
|f(x)|=\left|\frac{1}{2 \pi} \int_{\mathbb{R}} \mathcal{F}(f)(y) \mathrm{e}^{i x y} \mathrm{~d} y\right| \leq \frac{\nu_{\beta}}{\sqrt{2 \pi}}\left(\int_{\mathbb{R}}|\mathcal{F}(f)(y)|^{2}\left(1+|y|^{\beta}\right)^{2} \mathrm{~d} y\right)^{1 / 2} .
$$

We finish the proof by writing for $\mathcal{F}(f)$ continuous that

$$
\int_{\mathbb{R}}|\mathcal{F}(f)(y)|^{2}\left(1+|y|^{\beta}\right)^{2} \mathrm{~d} y \leq 4\left(\int_{|y| \leq 1}|\mathcal{F}(f)(y)|^{2} \mathrm{~d} y+\int_{|y|>1}|\mathcal{F}(f)(y)|^{2}|y|^{2 \beta} \mathrm{d} y\right)
$$

which is finite.

From now on, we suppose that $\beta$ is fixed, $\beta \in B, f$ belongs to the class $W_{n}(\beta, L)$ and $\gamma$ is also in $B$ such that $1 / 2<\gamma \leq \beta$. Let us define the nonrandom sequences

$$
h_{n, \gamma}=k_{\gamma}\left(\frac{f\left(x_{0}\right) \log n}{n}\right)^{\frac{1}{2 \gamma}} \text { and } \eta_{n, \gamma}=\nu_{\gamma} \sqrt{\frac{q}{2 \gamma k_{\gamma}}}\left(\frac{f\left(x_{0}\right) \log n}{n}\right)^{\frac{\gamma-1 / 2}{2 \gamma}} \text {. }
$$

Let $\delta_{n}=1 / \log n$ and let $1 / 2<\beta_{0} \leq 1$. Then (2.1) and (2.11) entail:

$$
\frac{n\left(h_{n} \delta_{n} \rho_{n}\right)^{2}}{\log n} \rightarrow \infty \text { and } \frac{h_{n}^{\beta_{0}-1 / 2}}{\delta_{n} \rho_{n}} \rightarrow 0
$$

We put for any $\gamma$ in $B$

$$
\widetilde{\gamma}=\left\{\begin{array}{c}
\beta, \text { when } \gamma=\beta \text { or } \gamma<\beta<2 \gamma \\
\gamma+\frac{\beta_{1}+1 / 2}{2}, \text { when } 2 \gamma \leq \beta
\end{array}\right.
$$

which satisfies $\widetilde{\gamma}<2 \gamma$ and $\gamma<\widetilde{\gamma} \leq \beta$. We define the random event

$$
A_{n, \gamma}=\left\{\left|\left(\frac{\widehat{h}_{n, \gamma}}{h_{n, \gamma}}\right)^{\bar{\gamma}-1 / 2}-1\right| \leq \delta_{n}\right\}
$$

and the corresponding nonrandom set

$$
\mathcal{H}_{n, \gamma}=\left\{h:\left|\left(\frac{h}{h_{n, \gamma}}\right)^{\bar{\gamma}-1 / 2}-1\right| \leq \delta_{n}\right\}
$$

The following result concerns the preliminary kernel estimator $\widehat{f}_{n}\left(x_{0}\right)$ in $(2.10)$, having the bandwidth $h_{n}$ that satisfies (2.11) and a bounded, kernel $K$, such that $\int|u||K(u)| \mathrm{d} u<\infty$. 
Lemma 3.2. If $\bar{A}_{n, \gamma}$ denotes the complementary set of $A_{n, \gamma}$, then for sufficiently small, fixed $\alpha>0$ :

$$
P_{f}\left[\bar{A}_{n, \gamma}\right] \leq 2 \exp \left\{-\frac{n\left((1-\alpha) h_{n} \delta_{n} \rho_{n}\right)^{2}}{2\|K\|_{\infty}^{2}}\right\}=o(1) .
$$

Proof. We use the facts that $\left|x^{\alpha}-1\right| \leq|x-1|$ for any $0<\alpha<1, x>0$ and $\left|\max \left\{x, \rho_{n}\right\}-\max \left\{y, \rho_{n}\right\}\right|$ $\leq|x-y|$ for any fixed $n$ and $x, y>0$. For $\alpha=\frac{\bar{\gamma}-1 / 2}{2 \gamma} \leq 1-\frac{1}{4 \beta_{1}}$ and $f\left(x_{0}\right) \geq \rho_{n}$, we have

$$
\begin{aligned}
P_{f}\left[\bar{A}_{n, \gamma}\right] & =P_{f}\left[\left|\left(\frac{\widehat{\rho}_{n}\left(x_{0}\right)}{f\left(x_{0}\right)}\right)^{\frac{\bar{\gamma}-1 / 2}{2 \gamma}}-1\right| \geq \delta_{n}\right] \leq P_{f}\left[\left|\widehat{f}_{n}\left(x_{0}\right)-f\left(x_{0}\right)\right| \geq \delta_{n} \rho_{n}\right] \\
& \leq P_{f}\left[\left|\widehat{f}_{n}\left(x_{0}\right)-E_{f} \widehat{f}_{n}\left(x_{0}\right)\right|+\left|E_{f} \widehat{f}_{n}\left(x_{0}\right)-f\left(x_{0}\right)\right| \geq \delta_{n} \rho_{n}\right] .
\end{aligned}
$$

By embedding Theorem 15.1 in Besov et al. [2] we deduce that $f$ which belongs to $W_{n}(\beta, L)$ belongs also to $W_{n}\left(\beta_{0}, L\right)$ with $\beta_{0}=\min \left\{\beta_{1}, 1\right\}$, which is included in the Hölder class $H\left(\beta_{0}-1 / 2, L_{0}\right), L_{0}>0$. This means $f$ verifies:

$$
|f(x)-f(y)| \leq L_{0}|x-y|^{\beta_{0}-1 / 2} .
$$

Let us consider a kernel $K$ such that $\int|u||K(u)| d u<\infty$. Then, we have:

$$
\begin{aligned}
\left|E_{f} \widehat{f}_{n}\left(x_{0}\right)-f\left(x_{0}\right)\right| & \leq \int|K(x)|\left|f\left(x_{0}+h_{n} x\right)-f\left(x_{0}\right)\right| \mathrm{d} x \\
& \leq L_{0} h_{n}^{\beta_{0}-1 / 2}\left(\int_{|x| \leq 1}|K(x)||x|^{\beta_{0}-1 / 2} \mathrm{~d} x+\int_{|x|>1}|K(x)||x| \mathrm{d} x\right) \\
& \leq c\left(L_{0}, \beta_{0}\right) h_{n}^{\beta_{0}-1 / 2},
\end{aligned}
$$

with a constant $c\left(L_{0}, \beta_{0}\right)>0$. The last term of the inequality above is equal to $o\left(\delta_{n} \rho_{n}\right)$ by (3.1). We obtain, for fixed, small $\alpha>0$

$$
P_{f}\left[\bar{A}_{n, \gamma}\right] \leq P_{f}\left[\left|\widehat{f}_{n}\left(x_{0}\right)-E_{f} \widehat{f}_{n}\left(x_{0}\right)\right| \geq(1-\alpha) \delta_{n} \rho_{n}\right] .
$$

We apply Hoeffding's inequality for the i.i.d. variables $K\left(\left(X_{i}-x_{0}\right) / h_{n}\right)$, bounded by $\|K\|_{\infty}<\infty$ :

$$
P_{f}\left[\left|\frac{1}{n} \sum_{i=1}^{n}\left(K\left(\frac{X_{i}-x_{0}}{h_{n}}\right)-E_{f} K\left(\frac{X_{i}-x_{0}}{h_{n}}\right)\right)\right| \geq z\right] \leq 2 \exp \left\{-\frac{n z^{2}}{2\|K\|_{\infty}^{2}}\right\}
$$

for any $z \geq 0, f \in W_{n}(\beta, L)$. It suffices to take $z=(1-\alpha) h_{n} \delta_{n} \rho_{n}$ in order to get the stated result. Moreover, $n\left(h_{n} \delta_{n} \rho_{n}\right)^{2} \rightarrow \infty$ with $n$, by (3.1), then the right-hand side in the lemma is $o(1)$.

Denote $f_{n, \gamma}\left(x_{0}, h\right)=\frac{1}{n h} \sum_{i=1}^{n} K_{\gamma}\left(\frac{X_{i}-x_{0}}{h}\right)$ the kernel estimator of $f\left(x_{0}\right)$, having kernel $K_{\gamma}$ and bandwidth $h$ in the set $\mathcal{H}_{n, \gamma}$. In particular, we denote $f_{n, \gamma}\left(x_{0}\right)=f_{n, \gamma}\left(x_{0}, h_{n, \gamma}\right)$.

We study in the following this kernel estimator using the classical decomposition

$$
\left|f_{n, \gamma}\left(x_{0}, h\right)-f\left(x_{0}\right)\right| \leq B_{n, \gamma}(h)+Z_{n, \gamma}(h),
$$

where $B_{n, \gamma}(h)=\left|E_{f} f_{n, \gamma}\left(x_{0}, h\right)-f\left(x_{0}\right)\right|$ is the bias of the estimator $f_{n, \gamma}\left(x_{0}, h\right)$ of $f\left(x_{0}\right)$, for a bandwidth $h \in$ $\mathcal{H}_{n, \gamma}$ and $Z_{n, \gamma}(h)=\left|f_{n, \gamma}\left(x_{0}, h\right)-E_{f} f_{n, \gamma}\left(x_{0}, h\right)\right|$ its stochastic term. 
Lemma 3.3. For any $h$ in $\mathcal{H}_{n, \gamma}, f \in W_{n}(\beta, L)$ and $\gamma, \beta$ in $B_{N_{n}}$ such that $1 / 2<\gamma \leq \beta$, let $\widetilde{L}>0$ be some constant, $b_{n, \gamma}(h)=\left\{\begin{array}{c}L b_{\beta} h^{\beta-\frac{1}{2}}, \gamma=\beta \\ \widetilde{L} \nu_{\max } h^{\bar{\gamma}-\frac{1}{2}}, \gamma<\beta\end{array}\right.$, where $\widetilde{\gamma}$ is defined by (3.2) and $s_{n, \gamma}^{2}(h)=f\left(x_{0}\right) \nu_{\gamma}^{2} /(n h)$. (In particular, we denote $b_{n, \gamma}=b_{n, \gamma}\left(h_{n, \gamma}\right)$ and $s_{n, \gamma}=s_{n, \gamma}\left(h_{n, \gamma}\right)$ ). We have:

1. $B_{n, \gamma}(h) \leq b_{n, \gamma}(h)$;

2. $E_{f}\left[Z_{n, \gamma}^{2}(h)\right] \leq s_{n, \gamma}^{2}(h)(1+o(1))$, where $o(1) \underset{n \rightarrow \infty}{\rightarrow} 0$ uniformly in $\gamma$ and $\beta$.

Proof. 1) We have $\mathcal{F}\left(\frac{1}{h} K_{\gamma}\left(\frac{-x_{0}}{h}\right)\right)(x)=\mathrm{e}^{i x x_{0}} \mathcal{F}\left(K_{\gamma}\right)(h x)$. Then

$$
\begin{aligned}
B_{n, \gamma}(h) & =\frac{1}{2 \pi}\left|\int_{\mathbb{R}} \mathcal{F}(f)(x) \mathrm{e}^{i x x_{0}}\left[\mathcal{F}\left(K_{\gamma}\right)(h x)-1\right] \mathrm{d} x\right| \leq \frac{1}{2 \pi} \int_{\mathbb{R}}|\mathcal{F}(f)(x)| \frac{|h x|^{2 \gamma}}{1+|h x|^{2 \gamma}} \mathrm{d} x \\
& \leq \frac{1}{\sqrt{2 \pi}}\left(\int_{\mathbb{R}} \frac{1}{2 \pi}|\mathcal{F}(f)(x)|^{2}|x|^{2 \widetilde{\gamma}} \mathrm{d} x\right)^{1 / 2}\left(\int_{\mathbb{R}} \frac{h^{2 \widetilde{\gamma}}|h x|^{2(2 \gamma-\tilde{\gamma})} \mathrm{d} x}{\left(1+|h x|^{2 \gamma}\right)^{2}}\right)^{1 / 2} .
\end{aligned}
$$

by the Cauchy-Schwarz inequality.

a) When $\gamma=\beta$ we have $\widetilde{\gamma}=\beta$ and

$$
B_{n, \beta}(h) \leq \frac{L h^{\beta-\frac{1}{2}}}{\sqrt{2 \pi}}\left(\int_{\mathbb{R}} \frac{|u|^{2 \beta}}{\left(1+|u|^{2 \beta}\right)^{2}} \mathrm{~d} u\right)^{1 / 2}=b_{n, \beta}(h) .
$$

b) When $\gamma<\beta$, we have $\widetilde{\gamma}$ such that $\gamma<\widetilde{\gamma}<2 \gamma$ and $\widetilde{\gamma} \leq \beta$. Then $f \in W_{n}(\beta, L) \subseteq W_{n}(\widetilde{\gamma}, \widetilde{L})$, for some fixed constant $\widetilde{L}>0$, by embedding theorem of Sobolev spaces. We get

$$
B_{n, \gamma}(h) \leq \frac{\widetilde{L} h^{\tilde{\gamma}-\frac{1}{2}}}{\sqrt{2 \pi}}\left(\int_{\mathbb{R}} \frac{|u|^{2(2 \gamma-\tilde{\gamma})}}{\left(1+|u|^{2 \gamma}\right)^{2}} \mathrm{~d} u\right)^{1 / 2} \leq b_{n, \gamma}(h) .
$$

2) We have $E_{f}\left[Z_{n, \gamma}^{2}(h)\right] \leq \frac{1}{n h} \int \frac{1}{h} K_{\gamma}^{2}\left(\frac{x-x_{0}}{h}\right) f(x) \mathrm{d} x$ and we conclude by Bochner's lemma applied to continuous $f$ and kernel $K_{\gamma}$, that

$$
\int \frac{1}{h} K_{\gamma}^{2}\left(\frac{x-x_{0}}{h}\right) f(x) \mathrm{d} x \leq f\left(x_{0}\right)\left\|K_{\gamma}\right\|_{2}^{2}(1+o(1)) .
$$

We give rough non-random upper bounds concerning the kernel estimator $\widehat{f}_{n, \gamma}\left(x_{0}\right)$ with bandwidth $\widehat{h}_{n, \gamma}$ depending on random data $X_{1}, \ldots, X_{n}$.

Lemma 3.4. For $\gamma<\beta$, there exist constants $d_{0}$ and $d_{1}$, such that, a.s.

$$
\sup _{\beta \in B} \sup _{f \in W_{n}(\beta, L)} \psi_{n, \beta}^{-q}\left(\widehat{\eta}_{n, \beta}+\left|\widehat{f}_{n, \beta}\left(x_{0}\right)-f\left(x_{0}\right)\right|\right)^{q} \leq 2 d_{0} n^{d_{1}}
$$

and

$$
\sup _{\beta \in B} \sup _{f \in W_{n}(\beta, L)} \psi_{n, \beta}^{-q}\left|\widehat{f}_{n, \gamma}\left(x_{0}\right)-f\left(x_{0}\right)\right|^{q} \leq d_{0} n^{d_{1}} .
$$


Proof. Let us bound at first the preliminary estimator $\left|\widehat{f}_{n}\left(x_{0}\right)\right| \leq\left\|K_{0}\right\|_{\infty} / h_{n}$. Then, a.s.

$$
\left|\widehat{f}_{n, \gamma}\left(x_{0}\right)\right| \leq \frac{\left\|K_{\gamma}\right\|_{\infty}}{\widehat{h}_{n, \gamma}} \leq \frac{K_{\max }}{k_{\min }}\left(\frac{n}{\widehat{\rho}_{n}\left(x_{0}\right) \log n}\right)^{\frac{1}{2 \gamma}} \leq O(1) n^{\frac{1}{2 \beta_{1}}}
$$

At last, for $\beta$ in $B_{-}$

$$
\psi_{n, \beta}^{-1} \leq \frac{\sqrt{2 \beta-1}}{2 \beta L \nu_{\beta} k_{\beta}^{\beta}} \sqrt{k_{\beta}}\left(\frac{n}{f\left(x_{0}\right) \log n}\right)^{\frac{1}{2}-\frac{1}{4 \beta}} \leq O(1) \sqrt{\beta_{N_{n}}} n^{\frac{1}{2}-\frac{1}{4 \beta_{1}}}
$$

and the same holds for $\beta=\beta_{N_{n}}, \psi_{n, \beta_{N_{n}}}^{-1} \leq O(1) n^{1 / 2-1 /\left(4 \beta_{1}\right)}$.

We may conclude that a.s., for any $1 / 2<\gamma \leq \beta$ :

$$
\psi_{n, \beta}^{-1}\left|\widehat{f}_{n, \gamma}\left(x_{0}\right)-f\left(x_{0}\right)\right| \leq O(1) \sqrt{\beta_{N_{n}}}\left(n^{\frac{1}{2}+\frac{1}{4 \beta_{1}}}+n^{\frac{1}{2}-\frac{1}{4 \beta_{1}}}\right) \leq d_{0} n^{d_{1}}
$$

since $\beta_{N_{n}}=o(1)$. Similarly,

$$
\frac{\widehat{\eta}_{n, \beta}}{\psi_{n, \beta}}=\frac{\eta_{n, \beta}}{\psi_{n, \beta}} \cdot\left(\frac{\widehat{\rho}_{n}\left(x_{0}\right)}{f\left(x_{0}\right)}\right)^{\frac{(\beta-1 / 2)}{2 \beta}} \leq\left(1-\frac{1}{2 \beta}\right)\left(\frac{\widehat{\rho}_{n}}{\rho_{n}}\right)^{\frac{1}{2}-\frac{1}{4 \beta}} \leq d_{0} n^{d_{1}}
$$

Let us choose a convenient sequence which shall appear in the proof of the upper bounds:

$$
\tau_{n, \gamma}=s_{n, \gamma}\left[\left[\frac{q}{2}\left(\frac{1}{\gamma}-\frac{1}{\beta}\right) \log n\right]^{\frac{1}{2}}+\left(\frac{\log n}{\beta_{N_{n}}}\right)^{\frac{1}{4}}\right], \text { for any } \gamma \leq \beta
$$

Remark that $\tau_{n, \beta} / s_{n, \beta}=\left(\log n / \beta_{N_{n}}\right)^{1 / 4} \rightarrow \infty$, when $n \rightarrow \infty$.

Lemma 3.5. 1. The following inequalities hold for any $\beta \in B_{n}$ and $f \in W_{n}(\beta, L)$

$$
\begin{aligned}
\frac{s_{n, \beta}}{\psi_{n, \beta}} & \leq \sqrt{\frac{2}{q}}\left(\frac{\beta_{N_{n}}}{\log n}\right)^{1 / 2}, \frac{\tau_{n, \beta}}{\psi_{n, \beta}} \leq \sqrt{\frac{2}{q}}\left(\frac{\beta_{N_{n}}}{\log n}\right)^{\frac{1}{4}}, \\
\left(\frac{\log n}{n}\right)^{\frac{\Delta_{n}}{\beta_{N}^{2}}} & \leq \exp \left\{-\frac{\Delta_{n} \log n}{\beta_{N}^{2} \log \log n}\right\}_{n \rightarrow \infty}^{\rightarrow} 0 .
\end{aligned}
$$

2. If $\gamma<\beta$ are in $B_{n}$ we have

$$
\sup _{f \in W_{n}(\beta, L)} \frac{b_{n, \gamma}^{q}+\tau_{n, \gamma}^{q}}{\psi_{n, \beta}^{q}} \leq O(1)(\log n)^{q / 2} n^{q\left(\frac{1}{4 \gamma}-\frac{1}{4 \beta}\right)}
$$

Proof. 1. It suffices to note that $\eta_{n, \beta}^{2}=\left(\frac{2 \beta-1}{2 \beta}\right)^{2} \psi_{n, \beta}^{2}=\frac{q}{2 \beta} s_{n, \beta}^{2} \log n$ and use (2.4) for the limit.

2. First, if $\beta$ is in $B_{-}$, we have

$$
\psi_{n, \beta}^{-1} \leq O(1) \sqrt{\beta_{N}}\left(\frac{n}{f\left(x_{0}\right) \log n}\right)^{\frac{1}{2}-\frac{1}{4 \beta}} \leq O(1) \sqrt{\log n}\left(\frac{n}{f\left(x_{0}\right) \log n}\right)^{\frac{1}{2}-\frac{1}{4 \beta}} .
$$


We see as well that

$$
\tau_{n, \gamma} \leq s_{n, \gamma} \sqrt{\frac{q \log n}{2 \gamma}}(1+o(1)) \leq O(1)\left(\frac{f\left(x_{0}\right) \log n}{n}\right)^{\frac{1}{2}-\frac{1}{4 \gamma}}
$$

and also

$$
b_{n, \gamma} \leq c_{b} h_{n, \gamma}^{\tilde{\gamma}-\frac{1}{2}} \leq O(1)\left(\frac{f\left(x_{0}\right) \log n}{n}\right)^{\frac{1}{2}-\frac{1}{4 \gamma}}
$$

as $\gamma \leq \widetilde{\gamma}<2 \gamma$ and $k_{\text {min }} \leq k_{\gamma} \leq k_{\max }$. Moreover, $f\left(x_{0}\right) \log n \geq \rho_{n} \log n>0$, then

$$
\sup _{f \in W_{n}(\beta, L)} \frac{b_{n, \gamma}^{q}+\tau_{n, \gamma}^{q}}{\psi_{n, \beta}^{q}} \leq O(1)(\log n)^{q / 2} n^{q\left(\frac{1}{4 \gamma}-\frac{1}{4 \beta}\right)}
$$

If $\beta=\beta_{N_{n}}$, the result is immediate.

\section{EXPonential inequalities AND APPliCATions}

The key lemmas in the proof of the upper bounds are given here and the results as we apply them in the next section are stated as theorems.

\subsection{Exponential bounds}

The next proposition recalls two inequalities on empirical processes. Denote the empirical distribution associated to the i.i.d. observations $X_{1}, \ldots, X_{n}$ of common law $P$ by $\mathbb{P}_{n}=1 / n \sum_{i=1}^{n} \delta_{X_{i}}$.

Proposition 4.1. Let us consider a class of functions $\mathcal{K}=\left\{f_{h}(\cdot) / h \in H\right\}$ satisfying

$$
\sup _{h \in H}\left\|f_{h}\right\|_{\infty} \leq K \text { and } \sup _{h \in H}\left\|f_{h}\right\|_{\mathbb{L}_{2}(P)} \leq M
$$

for some positive $K$ and $M$.

Then for all $u>0$ and for fixed $h \in H$

$$
P_{f}\left[\left|\int f_{h} \mathrm{~d}\left(\mathbb{P}_{n}-P\right)\right| \geq u\right] \leq 2 \exp \left\{-\frac{n u^{2}}{2\left(M^{2}+2 u K\right)}\right\}
$$

(Bernstein's Inequality see Pollard [29]);

Moreover, if

$$
u \leq \min \left\{8 M, \frac{2 C_{1} M^{2}}{K}\right\} \text { and } u \geq \frac{C_{0}}{\sqrt{n}} \int_{\frac{u}{2^{6}}}^{M} \max \left\{H_{B}^{1 / 2}(\mathcal{K}, x), 1\right\} \mathrm{d} x,
$$

where $H_{B}$ denotes the entropy with bracketing with respect to the $\mathbb{L}_{2}$-norm of a class of functions, then there exists some $a<1 / 2$ such that

$$
P_{f}\left[\sup _{h \in H}\left|\int f_{h} \mathrm{~d}\left(\mathbb{P}_{n}-P\right)\right| \geq u\right] \leq 8 \exp \left\{-a \frac{n u^{2}}{M^{2}}\right\}
$$

(Van de Geer [31]). 
One can prove without difficulty that there exists a positive constant $d_{2}=1 /\left(\beta_{1}-1 / 2\right)$, such that:

$$
\mathcal{H}_{n, \gamma} \subseteq\left\{h:\left|\frac{h}{h_{n, \gamma}}-1\right| \leq d_{2} \delta_{n}\right\}=H_{n, \gamma}
$$

We shall apply the previous results, successively, to two classes of measurable functions, for observations having distribution $P_{f}$, corresponding to the probability density $f \in W_{n}(\beta, L)$ and empirical distribution $\mathbb{P}_{n}$. Let us take on one hand

$$
\mathcal{K}=\left\{K_{\gamma, h}(\cdot)=\frac{1}{h} K_{\gamma}\left(\frac{\cdot-x_{0}}{h}\right): h \in H_{n, \gamma}\right\}
$$

Then

$$
\sup _{h \in H_{n, \gamma}}\left\|K_{\gamma, h}\right\|_{\infty} \leq \frac{\left\|K_{\gamma}\right\|_{\infty}}{h_{n, \gamma}} \leq \frac{K_{\max }}{h_{n, \gamma}}
$$

and by Lemma 3.3

$$
\sup _{h \in H_{n, \gamma}}\left\|K_{\gamma, h}\right\|_{\mathbb{L}_{2}\left(P_{f}\right)} \leq \frac{\nu_{\gamma} \sqrt{f\left(x_{0}\right)}}{\sqrt{h_{n, \gamma}}}=s_{n, \gamma} \sqrt{n} .
$$

For different $h_{1}, h_{2}$ in $H_{n, \gamma}$ we have

$$
\begin{aligned}
\left\|K_{\gamma, h_{1}}-K_{\gamma, h_{2}}\right\|_{\mathbb{L}_{2}\left(P_{f}\right)} & \leq \frac{1}{\sqrt{2 \pi}}\left\|\mathcal{F}\left(K_{\gamma}\right)\left(h_{1} \cdot\right)-\mathcal{F}\left(K_{\gamma}\right)\left(h_{2} \cdot\right)\right\|_{2} \\
& \leq \frac{1}{\sqrt{2 \pi}}\left(\int \frac{\left|h_{1}^{2 \gamma}-h_{2}^{2 \gamma}\right|^{2}|y|^{4 \gamma} \mathrm{d} y}{\left(1+\left|h_{1} y\right|^{2 \gamma}\right)^{2}\left(1+\left|h_{2} y\right|^{2 \gamma}\right)^{2}}\right)^{1 / 2} \\
& \leq \frac{O(1)}{\sqrt{h_{n, \gamma}}}\left|1-\left(\frac{h_{1}}{h_{2}}\right)^{2 \gamma}\right|
\end{aligned}
$$

Then, we may write that the $\varepsilon$-entropy with bracketing of the class $\mathcal{K}$ with respect to the $\mathbb{L}_{2}\left(P_{f}\right)$ norm is: $H_{B}(\mathcal{K}, \varepsilon) \leq O\left(\log \frac{1}{\varepsilon}+\log n\right)$. Thus

$$
\frac{C_{0}}{\sqrt{n}} \int_{0}^{M} \max \left(H_{B}^{1 / 2}(\mathcal{K}, x), 1\right) \mathrm{d} x \leq M \sqrt{\frac{\log n}{n}}
$$

On the other hand, consider the following class of measurable functions

$$
\mathcal{K}\left(h_{n, \gamma}\right)=\left\{K_{\gamma, h}(\cdot)-K_{\gamma, h_{n, \gamma}}(\cdot): h \in H_{n, \gamma}\right\}
$$


for which

$$
\begin{aligned}
\sup _{h \in H_{n, \gamma}}\left\|K_{\gamma, h}-K_{\gamma, h_{n, \gamma}}\right\|_{\infty} & \leq \sup _{h \in H_{n, \gamma}} \sup _{x} \frac{1}{\sqrt{2 \pi}}\left|\int \mathcal{F}\left(K_{\gamma, h}-K_{\gamma, h_{n, \gamma}}\right)(y) \mathrm{e}^{i x y} \mathrm{~d} y\right| \\
& \leq \sup _{h \in H_{n, \gamma}} \frac{1}{\sqrt{2 \pi}} \int \frac{\left|h^{2 \gamma}-h_{n, \gamma}^{2 \gamma}\right||y|^{2 \gamma} \mathrm{d} y}{\left(1+|h y|^{2 \gamma}\right)\left(1+\left|h_{n, \gamma} y\right|^{2 \gamma}\right)} \\
& \leq \frac{O(1)}{h_{n, \gamma}}\left|1-\left(\frac{h}{h_{n, \gamma}}\right)^{2 \gamma}\right| \leq O(1) \frac{\beta_{N} \delta_{n}}{h_{n, \gamma}}
\end{aligned}
$$

and

$$
\sup _{h \in H_{n, \gamma}}\left\|K_{\gamma, h}-K_{\gamma, h_{n, \gamma}}\right\|_{\mathbb{L}_{2}\left(P_{f}\right)} \leq \sup _{h \in H_{n, \gamma}} \frac{O(1)}{\sqrt{h_{n, \gamma}}}\left|1-\left(\frac{h}{h_{n, \gamma}}\right)^{2 \gamma}\right| \leq O(1) \frac{\beta_{N} \delta_{n}}{\sqrt{h_{n, \gamma}}}
$$

It is easy to see, that (4.2) still holds for this class.

Lemma 4.2. For the kernel estimator $f_{n, \gamma}\left(x_{0}, h\right)$, for any fixed $x_{0}$ and for $u \leq c_{1} s_{n, \gamma} \sqrt{\log n}\left(c_{1}>0\right.$, an absolute constant), there exists $\delta_{n 0}>0$ small enough, independent of $\gamma$, such that:

$$
P_{f}\left[\left|Z_{n, \gamma}\left(h_{n, \gamma}\right)\right| \geq u\right] \leq 2 \exp \left\{-\frac{u^{2}}{2 s_{n, \gamma}^{2}}\left(1-\delta_{n 0}\right)\right\} .
$$

Proof. Write $Z_{n, \gamma}(h)=\int K_{\gamma, h} \mathrm{~d}\left(\mathbb{P}_{n}-P_{f}\right)$ and apply Bernstein's inequality of Proposition 4.1 for $K=K_{\max } / h_{n, \gamma}$ and $M=s_{n, \gamma} \sqrt{n}$ to get

$$
P_{f}\left[\left|Z_{n, \gamma}\left(h_{n, \gamma}\right)\right| \geq u\right] \leq 2 \exp \left\{-\frac{u^{2}}{2 s_{n, \gamma}^{2}\left(1+2 u K_{\max } / \nu_{\gamma}^{2} f\left(x_{0}\right)\right)}\right\} .
$$

We remark that for $u \leq c_{1} s_{n, \gamma} \sqrt{\log n}\left(c_{1}>0\right)$ we have

$$
\frac{2 u K_{\max }}{\nu_{\gamma}^{2} f\left(x_{0}\right)} \leq \frac{2 c_{1} K_{\max } \sqrt{\log n}}{\nu_{\gamma} \sqrt{f\left(x_{0}\right)} \sqrt{n h_{n, \gamma}}} \leq O(1)\left(\frac{\rho_{n} \log n}{n}\right)^{\frac{1}{2}-\frac{1}{4 \gamma}}=o(1)
$$

and thus we can find a $\delta_{n 0}>0$ small enough, such that

$$
\left(1+\frac{2 u K_{\max }}{\nu_{\gamma}^{2} f\left(x_{0}\right)}\right) \leq \frac{1}{1-\delta_{n 0}}
$$

which we can replace in the exponential inequality above in order to get the stated lemma.

Lemma 4.3. For $u$, such that $\tau_{n, \gamma} \leq u \leq c_{1} s_{n, \gamma} \sqrt{\log n}$, there exist a constant $d_{3}>0$ and $\delta_{n 1}>0$ sufficiently small, independent of $\gamma$, such that

$$
P_{f}\left[\sup _{h \in H_{n, \gamma}}\left|Z_{n, \gamma}(h)\right| \geq u\right] \leq d_{3} \exp \left\{-\frac{u^{2}\left(1-\delta_{n 1}\right)^{2}}{2 s_{n, \gamma}^{2}}\left(1-\delta_{n 0}\right)\right\},
$$

for $n$ large enough, where $\delta_{n 0}$ is the sequence in Lemma 4.2. 
Proof. Let us consider the sequence $\delta_{n 1}=\beta_{N_{n}} \delta_{n} \sqrt{\beta_{N_{n}} \log n} \underset{n \rightarrow \infty}{\rightarrow} 0$. We have

$$
\begin{aligned}
P_{f}\left[\sup _{h \in H_{n, \gamma}}\left|Z_{n, \gamma}(h)\right| \geq u\right] \leq & P_{f}\left[\left|Z_{n, \gamma}\left(h_{n, \gamma}\right)\right| \geq u\left(1-\delta_{n 1}\right)\right] \\
& +P_{f}\left[\sup _{h \in H_{n, \gamma}}\left|Z_{n, \gamma}(h)-Z_{n, \gamma}\left(h_{n, \gamma}\right)\right| \geq u \delta_{n 1}\right] .
\end{aligned}
$$

We apply Lemma 4.2 to the first term on the right-hand side of the previous inequality. We see that $u\left(1-\delta_{n 1}\right)$ $\leq u \leq c_{1} s_{n, \gamma} \sqrt{\log n}$ and thus there exist a sufficiently small $\delta_{n 0}>0$ such that:

$$
P_{f}\left[\left|Z_{n, \gamma}\left(h_{n, \gamma}\right)\right| \geq u\left(1-\delta_{n 1}\right)\right] \leq 2 \exp \left\{-\frac{u^{2}\left(1-\delta_{n 1}\right)^{2}}{2 s_{n, \gamma}^{2}}\left(1-\delta_{n 0}\right)\right\} .
$$

For the second term we first see that

$$
Z_{n, \gamma}(h)-Z_{n, \gamma}\left(h_{n, \gamma}\right)=\int\left(K_{\gamma, h}-K_{\gamma, h_{n, \gamma}}\right) \mathrm{d}\left(\mathbb{P}_{n}-P_{f}\right) .
$$

Then we apply Van de Geer's inequality from Proposition 4.1 , where $K=\beta_{N_{n}} \delta_{n} / h_{n, \gamma}$ and $M=\beta_{N_{n}} \delta_{n} / \sqrt{h_{n, \gamma}}$

$$
P_{f}\left[\sup _{h \in H_{n, \gamma}}\left|Z_{n, \gamma}(h)-Z_{n, \gamma}\left(h_{n, \gamma}\right)\right| \geq u \delta_{n 1}\right] \leq 8 \exp \left\{-a n h_{n, \gamma} \frac{\left(u \delta_{n 1}\right)^{2}}{\left(\beta_{N_{n}} \delta_{n}\right)^{2}}\right\}
$$

for all $u$ in $\left[\tau_{n, \gamma}, c_{1} s_{n, \gamma} \sqrt{\log n}\right]$. Indeed, $\min \left\{8 M, \frac{2 C_{1} M^{2}}{K}\right\}=O(1) \beta_{N_{n}} \delta_{n}$

$$
\frac{C_{0}}{\sqrt{n}} \int_{0}^{M} \max \left\{H_{B}^{1 / 2}(\mathcal{K}, x), 1\right\} \mathrm{d} x \leq O(1) \frac{\beta_{N_{n}} \delta_{n}}{\sqrt{n h_{n, \gamma}}} \sqrt{\log n}
$$

and

$$
\left[\tau_{n, \gamma} \delta_{n 1}, c_{1} \delta_{n 1} s_{n, \gamma} \sqrt{\log n}\right] \subseteq\left[\frac{\beta_{N_{n}} \delta_{n}}{\sqrt{n h_{n, \gamma}}} \sqrt{\log n}, \beta_{N_{n}} \delta_{n}\right]
$$

for $n$ large enough. By summing the two terms again, we get

$$
\begin{aligned}
P_{f}\left[\sup _{h \in H_{n, \gamma}}\left|Z_{n, \gamma}(h)\right| \geq u\right] & \leq 2 \exp \left\{-\frac{u^{2}\left(1-\delta_{n 1}\right)^{2}}{2 s_{n, \gamma}^{2}}\left(1-\delta_{n 0}\right)\right\}+8 \exp \left\{-a n h_{n, \gamma} \frac{\left(u \delta_{n 1}\right)^{2}}{\left(\beta_{N_{n}} \delta_{n}\right)^{2}}\right\} \\
& \leq 8(1+o(1)) \exp \left\{-\frac{u^{2}\left(1-\delta_{n 1}\right)^{2}}{2 s_{n, \gamma}^{2}}\left(1-\delta_{n 0}\right)\right\} .
\end{aligned}
$$

Theorem 4.4. We have

$$
\sup _{\beta \in B_{n}} \sup _{f \in W_{n}(\beta, L)} P_{f}\left[\sup _{h \in H_{n, \beta}}\left|Z_{n, \beta}(h)\right| \geq \tau_{n, \beta}\right] \underset{n \rightarrow \infty}{\rightarrow} 0 .
$$


Proof. Let us apply the Lemma 4.3 , for $\gamma=\beta, u=\tau_{n, \beta}$ and $\delta_{n 2} \underset{n \rightarrow \infty}{\rightarrow} 0$ such that $\left(1-\delta_{n 1}\right)^{2}\left(1-\delta_{n 0}\right)=1-\delta_{n 2}$

$$
P_{f}\left[\sup _{h \in H_{n, \beta}}\left|Z_{n, \beta}(h)\right| \geq \tau_{n, \beta}\right] \leq d_{3} \exp \left\{-\frac{\tau_{n, \beta}^{2}}{2 s_{n, \beta}^{2}}\left(1-\delta_{n 2}\right)\right\} \leq d_{3} \exp \left\{-\frac{1}{2}\left(\frac{\log n}{\beta_{N_{n}}}\right)^{\frac{1}{2}}\left(1-\delta_{n 2}\right)\right\}
$$

and this sequence tends to 0 , by Lemma 3.5 , uniformly in $f \in W_{n}(\beta, L)$ and $\beta \in B_{n}$.

Lemma 4.5. Let $c_{2}>0$ be a constant such that $c_{2} \geq c_{1} s_{n, \gamma} \sqrt{\log n}$. For $n$ large enough and $c_{1} s_{n, \gamma} \sqrt{\log n} \leq$ $u \leq c_{2}$, we have that

$$
P_{f}\left[\sup _{h \in H_{n, \gamma}}\left|Z_{n, \gamma}(h)\right| \geq u\right] \leq 8 \exp \left\{-a \frac{u^{2}}{s_{n, \gamma}^{2}}\right\} .
$$

Proof. Let us note that $Z_{n, \gamma}(h)=\int K_{\gamma, h} \mathrm{~d}\left(\mathbb{P}_{n}-P_{f}\right)$ and apply Van de Geer's inequality of Proposition 4.1, for $K=K_{\max } / h_{n, \gamma}$ and $M=s_{n, \gamma} \sqrt{n}$. Indeed,

$$
\min \left\{8 M, \frac{2 C_{1} M^{2}}{K}\right\}=\frac{2 C_{1} M^{2}}{K} \leq c_{2}
$$

for $c_{2}$ large enough, and

$$
\frac{C_{0}}{\sqrt{n}} \int_{0}^{M} \max \left\{H_{B}^{1 / 2}(\mathcal{K}, x), 1\right\} \mathrm{d} x \leq C_{0} \frac{M \sqrt{\log n}}{\sqrt{n}} \leq c_{1} s_{n, \gamma} \sqrt{\log n}
$$

The hypothesis are verified, then

$$
P_{f}\left[\sup _{h \in H_{n, \gamma}}\left|Z_{n, \gamma}(h)\right| \geq u\right] \leq 8 \exp \left\{-a \frac{n u^{2}}{M^{2}}\right\} \leq 8 \exp \left\{-a \frac{u^{2}}{s_{n, \gamma}^{2}}\right\} .
$$

Theorem 4.6. We have

$$
\sup _{\substack{\gamma \in B_{-} \\ \gamma<\beta}} \frac{\beta_{N_{n}}}{\Delta_{n}} \sup _{f \in W_{n}(\beta, L)} \psi_{n, \beta}^{-q} E_{f}\left[\left(\sup _{h \in H_{n, \gamma}}\left|Z_{n, \gamma}(h)\right|\right)^{q} I\left(\sup _{h \in H_{n, \gamma}}\left|Z_{n, \gamma}(h)\right| \geq \tau_{n, \gamma}\right)\right] \underset{n \rightarrow \infty}{\rightarrow} 0,
$$

uniformly in $\beta \in B_{n}$. In particular, when $\gamma=\beta$, we have

$$
\sup _{f \in W_{n}(\beta, L)} \psi_{n, \beta}^{-q} E_{f}\left[\left(\sup _{h \in H_{n, \beta}}\left|Z_{n, \beta}(h)\right|\right)^{q} I\left(\sup _{h \in H_{n, \beta}}\left|Z_{n, \beta}(h)\right| \geq \tau_{n, \beta}\right)\right] \underset{n \rightarrow \infty}{\rightarrow} 0
$$

uniformly in $\beta \in B_{n}$.

Proof. Let us write the expectation as follows:

$$
E_{f}\left[\left(\sup _{h \in H_{n, \gamma}}\left|Z_{n, \gamma}(h)\right|\right)^{q} I\left(\sup _{h \in H_{n, \gamma}}\left|Z_{n, \gamma}(h)\right| \geq \tau_{n, \gamma}\right)\right]=\int_{\tau_{n, \gamma}}^{c_{3} / h_{n, \gamma}} P_{f}\left[\sup _{h \in H_{n, \gamma}}\left|Z_{n, \gamma}(h)\right| \geq u\right] \mathrm{d} u^{q} .
$$


Indeed, we can bound $\sup _{h \in H_{n, \gamma}}\left|Z_{n, \gamma}(h)\right| \leq c_{3} / h_{n, \gamma}$ for some $c_{3}>0$ and it is useless to integrate further on. We split the integration domain into three intervals: $I=\left[\tau_{n, \gamma}, c_{1} s_{n, \gamma} \sqrt{\log n}\right], I I=\left[c_{1} s_{n, \gamma} \sqrt{\log n}, c_{2}\right]$ and $I I I=\left[c_{2}, c_{3} / h_{n, \gamma}\right]$, which are non-empty for $n$ large enough. Let us apply Lemma 4.3 on the interval $I$ and get

$$
\begin{aligned}
\int_{I} P_{f}\left[\sup _{h \in H_{n, \gamma}}\left|Z_{n, \gamma}(h)\right| \geq u\right] \mathrm{d} u^{q} & \leq d_{3} \int_{I} \exp \left\{-\frac{u^{2}}{2 s_{n, \gamma}^{2}}\left(1-\delta_{n 2}\right)\right\} \mathrm{d} u^{q} \\
& \leq O(1) s_{n, \gamma}^{q} \exp \left\{-\frac{\tau_{n, \gamma}^{2}}{2 s_{n, \gamma}^{2}}\left(1-\delta_{n 2}\right)\right\}
\end{aligned}
$$

Now

$$
\begin{aligned}
\psi_{n, \beta}^{-q} \int_{I} & \leq O(1) \frac{s_{n, \gamma}^{q}}{\psi_{n, \beta}^{q}} \exp \left\{-\left(\frac{q}{4}\left(\frac{1}{\gamma}-\frac{1}{\beta}\right) \log n+\frac{1}{2} \sqrt{\frac{\log n}{\beta_{N_{n}}}}\right)\left(1-\delta_{n 2}\right)\right\} \\
& \leq O(1) \exp \left\{-\frac{1}{2} \sqrt{\frac{\log n}{\beta_{N_{n}}}}\left(1-\delta_{n 2}\right)\right\}=o(1) .
\end{aligned}
$$

On the second interval $I I$, we write

$$
\begin{aligned}
\int_{I I} P_{f}\left[\sup _{h \in H_{n, \gamma}}\left|Z_{n, \gamma}(h)\right| \geq u\right] \mathrm{d} u^{q} & \leq \int_{I I} P_{f}\left[\sup _{h \in H_{n, \gamma}}\left|Z_{n, \gamma}(h)\right| \geq c_{1} s_{n, \gamma} \sqrt{\log n}\right] \mathrm{d} u^{q} \\
& \leq c_{2}^{q} P_{f}\left[\sup _{h \in H_{n, \gamma}}\left|Z_{n, \gamma}(h)\right| \geq c_{1} s_{n, \gamma} \sqrt{\log n}\right] \\
& \leq 8 c_{2}^{q} \exp \left\{-a c_{1}^{2} \log n\right\}
\end{aligned}
$$

by Lemma 4.5 and

$$
\begin{aligned}
\psi_{n, \beta}^{-q} \int_{I I} & \leq O(1) n^{\frac{q}{2}-\frac{q}{4 \beta}} \exp \left\{-a c_{1}^{2} \log n\right\} \\
& \leq O(1) \exp \left\{-\log n\left(a c_{1}^{2}-\frac{q}{2}+\frac{q}{4 \beta}\right)\right\} \\
& \leq O(1) \exp \left\{-q \frac{\log n}{4 \beta_{N_{n}}}-\log n\left(a c_{1}^{2}-\frac{q}{2}\right)\right\}=o(1)
\end{aligned}
$$

by choosing $c_{1}$ sufficiently large. Similarly,

$$
\begin{aligned}
\int_{I I I} P_{f}\left[\sup _{h \in H_{n, \gamma}}\left|Z_{n, \gamma}(h)\right| \geq u\right] \mathrm{d} u^{q} & \leq \int_{I I I} P_{f}\left[\sup _{h \in H_{n, \gamma}}\left|Z_{n, \gamma}(h)\right| \geq c_{2}\right] \mathrm{d} u^{q} \\
& \leq \frac{K_{\max }^{q}}{s_{n, \gamma}^{q}} \exp \left\{-a \frac{c_{2}^{2}}{s_{n, \gamma}^{2}}\right\} \\
& \leq \frac{O(1)}{s_{n, \gamma}^{q}} \exp \left\{-O(1) n^{1-\frac{1}{2 \beta_{1}}}\right\} .
\end{aligned}
$$


Now,

$$
\begin{aligned}
\psi_{n, \beta}^{-q} \int_{I I I} & \leq O(1) n^{q-\frac{q}{4 \beta}-\frac{q}{4 \gamma}} \exp \left\{-O(1) n^{1-\frac{1}{2 \beta_{1}}}\right\} \\
& \leq O(1) \exp \left\{-O(1) n^{1-\frac{1}{2 \beta_{1}}}+q\left(1-\frac{1}{2 \beta_{N_{n}}}\right) \log n\right\}=o(1)
\end{aligned}
$$

To finish the proof it suffices to remember relations (2.5) to (2.7) and see that the three sequences do not depend on $f$ in $W_{n}(\beta, L)$, nor on $\gamma$ and $\beta$ in $B_{n}$.

\subsection{Probability of undershooting}

We want to bound here the probability that the estimated value $\hat{\beta}$ is strictly less than the true value $\beta$ i.e. $P_{f}[\hat{\beta}=\gamma]$ for $\gamma$ in $(1 / 2, \beta)$ for the worst density in $W_{n}(\beta, L)$ and uniformly in $\beta$ over $B_{n}$. We show in fact that $\widehat{\beta}$ typically overestimates the true value $\beta$ and that the undershooting is a rare event.

Lemma 4.7. Let us choose the sequence $\delta_{n 3}=\exp \left\{-\frac{1}{8} \cdot \frac{\Delta_{n} \log n}{\beta_{N}^{2}}\right\}$ which tends to 0 , by condition (2.4). Then for any $\gamma_{0}$, $\gamma$ two elements of the set $B_{-}$such that $\gamma_{0} \leq \gamma<\beta$, we have for $\gamma_{1}=\min \left\{\alpha \in B_{N_{n}}: \alpha>\gamma\right\}$, $\gamma<\gamma_{1} \leq \beta$ and

$$
\frac{b_{n, \gamma_{0}}}{\delta_{n 3} \eta_{n, \gamma_{0}}} \underset{n \rightarrow \infty}{\rightarrow} 0, \frac{b_{n, \gamma_{1}}}{\delta_{n 3} \eta_{n, \gamma_{0}}} \underset{n \rightarrow \infty}{\rightarrow} 0, \frac{\delta_{n 3} s_{n, \gamma_{0}}}{s_{n, \gamma_{1}}} \underset{n \rightarrow \infty}{\rightarrow} \infty
$$

Proof. We see that for $\gamma_{0}<\gamma_{1}$ in $B_{-}: h_{n, \gamma_{0}} / h_{n, \gamma_{1}} \leq O(1)(\log n / n)^{\frac{\Delta_{n}}{2 \beta_{N}}} \leq o(1)$ by Lemma (3.5). We write

$$
\begin{aligned}
\frac{b_{n, \gamma_{0}}}{\delta_{n 3} \eta_{n, \gamma_{0}}} & =\sqrt{\frac{2 \gamma_{0}}{q \log n}} \frac{c_{b} h_{n, \gamma_{0}}^{\tilde{\gamma}_{0}-\frac{1}{2}}}{\delta_{n 3} s_{n, \gamma_{0}}} \leq O(1) \sqrt{\beta_{N}} \frac{h_{n, \gamma_{0}}^{\tilde{\gamma}_{0}-\gamma_{0}}}{\delta_{n 3}} \\
& \leq O(1) \sqrt{\beta_{N}} \exp \left\{\frac{1}{8} \frac{\Delta_{n} \log n}{\beta_{N}^{2}}-\frac{\left(\widetilde{\gamma}_{0}-\gamma_{0}\right) \log n}{2 \beta_{N}}\right\} .
\end{aligned}
$$

By definition of $\widetilde{\gamma}_{0}$, see $(3.2), \widetilde{\gamma}_{0}-\gamma_{0}>\min \left\{\beta-\gamma, \frac{1}{2}\right\} \geq \min \left\{\Delta_{n}, \frac{1}{2}\right\}>0$. Then

$$
\frac{b_{n, \gamma_{0}}}{\delta_{n 3} \eta_{n, \gamma_{0}}} \leq O(1) \exp \left\{-\frac{\log n}{2 \beta_{N}}\left(\min \left\{\Delta_{n}, \frac{1}{2}\right\}-\frac{\Delta_{n}}{4 \beta_{N}}-\frac{\log \beta_{N}}{2} \frac{\beta_{N}}{\log n}\right)\right\}
$$

which is an $o(1)$. It is easy to see now, that

$$
\frac{b_{n, \gamma_{1}}}{\delta_{n 3} \eta_{n, \gamma_{0}}}=\sqrt{\frac{2 \gamma_{0}}{q \log n}} \frac{c_{b} h_{n, \gamma_{1}}^{\tilde{\gamma}_{1}-\frac{1}{2}}}{\delta_{n 3} s_{n, \gamma_{0}}} \leq O(1) \frac{\sqrt{\beta_{N}}}{\delta_{n 3}} \sqrt{\frac{h_{n, \gamma_{0}}}{h_{n, \gamma_{1}}}} \leq O(1) \exp \left\{-\frac{1}{4} \frac{\Delta_{n} \log n}{\beta_{N}^{2}}\left(1-\frac{4 \log \beta_{N}}{\Delta_{n} \log n} \beta_{N}^{2}\right)\right\}
$$

which tends to 0 , for $\widetilde{\gamma}_{1}$ defined as in $(3.2)$. 
We conclude with the third limit, using also Lemma (3.5)

$$
\begin{aligned}
\frac{\delta_{n 3} s_{n, \gamma_{0}}}{s_{n, \gamma_{1}}} & =\delta_{n 3} \frac{\nu_{\gamma_{0}}}{\nu_{\gamma_{1}}} \sqrt{\frac{h_{n, \gamma_{1}}}{h_{n, \gamma_{0}}}} \geq O\left(\delta_{n 3}\right) n^{\frac{1}{4}\left(\frac{1}{\gamma_{0}}-\frac{1}{\gamma_{1}}\right)} \\
& \geq O(1) \exp \left\{\frac{1}{8} \frac{\Delta_{n} \log n}{\beta_{N}^{2}}\right\} \underset{n \rightarrow \infty}{\rightarrow} \infty
\end{aligned}
$$

Lemma 4.8. Let $\gamma, \beta$ in $B_{N_{n}}, \gamma<\beta$. Then, for the estimator $\hat{\beta}$ of $\beta$, there exists a constant $d_{4}>0$ such that:

$$
\sup _{\beta \in B_{N_{n}}} \sup _{f \in W_{n}(\beta, L)} P_{f}[\hat{\beta}=\gamma] \leq d_{4} \frac{\beta_{N}}{\Delta_{n}} n^{-\frac{q}{4 \gamma}}
$$

Proof. By the definition of $\hat{\beta}$, when the event $\{\hat{\beta}=\gamma\}$ occurs, then for $\gamma_{1}=\min \left\{\alpha \in B_{N_{n}}: \alpha>\gamma\right\}$, there exists at least one $\gamma_{0}, \gamma_{0}<\gamma_{1} \leq \beta$, for which

$$
\left|\widehat{f}_{n, \gamma_{1}}\left(x_{0}\right)-\widehat{f}_{n, \gamma_{0}}\left(x_{0}\right)\right|>\widehat{\eta}_{n, \gamma_{0}}
$$

Then

$$
\begin{aligned}
P_{f}[\hat{\beta}=\gamma] & \leq \sum_{\substack{\gamma_{0} \leq \gamma \\
\gamma_{0} \in B_{-}}} P_{f}\left[\left|\widehat{f}_{n, \gamma_{1}}\left(x_{0}\right)-\widehat{f}_{n, \gamma_{0}}\left(x_{0}\right)\right|>\widehat{\eta}_{n, \gamma_{0}}\right] \\
& \leq \operatorname{card} B_{-} \cdot \sup _{\substack{\gamma_{0} \leq \gamma \\
\gamma_{0} \in B_{-}}} P_{f}\left[\left|\widehat{f}_{n, \gamma_{1}}\left(x_{0}\right)-\widehat{f}_{n, \gamma_{0}}\left(x_{0}\right)\right|>\widehat{\eta}_{n, \gamma_{0}}\right] \\
& \leq \frac{\beta_{N}}{\Delta_{n}} \cdot \sup _{\substack{\gamma_{0} \leq \gamma \\
\gamma_{0} \in B_{-}}}\left(p_{1}+p_{2}\right)
\end{aligned}
$$

where

$$
\begin{aligned}
& p_{1}=P_{f}\left[\left\{\left|\widehat{f}_{n, \gamma_{1}}\left(x_{0}\right)-\widehat{f}_{n, \gamma_{0}}\left(x_{0}\right)\right|>\widehat{\eta}_{n, \gamma_{0}}\right\} \cap\left\{A_{n, \gamma_{0}} \cap A_{n, \gamma_{1}}\right\}\right], \\
& p_{2}=P_{f}\left[\left\{\left|\widehat{f}_{n, \gamma_{1}}\left(x_{0}\right)-\widehat{f}_{n, \gamma_{0}}\left(x_{0}\right)\right|>\widehat{\eta}_{n, \gamma_{0}}\right\} \cap\left\{\bar{A}_{n, \gamma_{0}} \cup \bar{A}_{n, \gamma_{1}}\right\}\right] .
\end{aligned}
$$

In the case where the event $A_{n, \gamma_{0}}$ holds, we have that

$$
\widehat{\eta}_{n, \gamma_{0}}=\eta_{n, \gamma_{0}}\left(\frac{\widehat{h}_{n, \gamma_{0}}}{h_{n, \gamma_{0}}}\right)^{\widetilde{\gamma_{0}}-1 / 2} \geq \eta_{n, \gamma_{0}}\left(1-\delta_{n}\right)
$$

and we may replace:

$$
p_{1} \leq P_{f}\left[\sup _{h \in \mathcal{H}_{n, \gamma_{1}}}\left|f_{n, \gamma_{1}}(h)-f\right|+\sup _{h \in \mathcal{H}_{n, \gamma_{0}}}\left|f_{n, \gamma_{0}}(h)-f\right|>\eta_{n, \gamma_{0}}\left(1-\delta_{n}\right)\right]
$$


Let us write that

$$
\begin{aligned}
& \sup _{h \in \mathcal{H}_{n, \gamma_{1}}}\left|f_{n, \gamma_{1}}(h)-f\right|+\sup _{h \in \mathcal{H}_{n, \gamma_{0}}}\left|f_{n, \gamma_{0}}(h)-f\right| \\
& \leq \sup _{h \in \mathcal{H}_{n, \gamma_{1}}}\left(B_{n, \gamma_{1}}(h)+\left|Z_{n, \gamma_{1}}(h)\right|\right)+\sup _{h \in \mathcal{H}_{n, \gamma_{0}}}\left(B_{n, \gamma_{0}}(h)+\left|Z_{n, \gamma_{0}}(h)\right|\right) \\
& \leq\left(b_{n, \gamma_{1}}+b_{n, \gamma_{0}}\right)\left(1+\delta_{n}\right)+\sup _{h \in \mathcal{H}_{n, \gamma_{1}}}\left|Z_{n, \gamma_{1}}(h)\right|+\sup _{h \in \mathcal{H}_{n, \gamma_{0}}}\left|Z_{n, \gamma_{0}}(h)\right| .
\end{aligned}
$$

By Lemma $4.7,\left(b_{n, \gamma_{1}}+b_{n, \gamma_{0}}\right)\left(1+\delta_{n}\right) \leq d_{5} \delta_{n 3} \eta_{n, \gamma_{0}}\left(1-\delta_{n}\right)$, for some $d_{5}>0$ and for $n$ large enough. Then, we replace

$$
\begin{aligned}
p_{1} \leq & P_{f}\left[\sup _{h \in \mathcal{H}_{n, \gamma_{1}}}\left|Z_{n, \gamma_{1}}(h)\right|+\sup _{h \in \mathcal{H}_{n, \gamma_{0}}}\left|Z_{n, \gamma_{0}}(h)\right| \geq \eta_{n, \gamma_{0}}\left(1-\delta_{n}\right)\left(1-d_{5} \delta_{n 3}\right)\right] \\
\leq & P_{f}\left[\sup _{h \in H_{n, \gamma_{0}}}\left|Z_{n, \gamma_{0}}(h)\right| \geq \eta_{n, \gamma_{0}}\left(1-\delta_{n}\right)\left(1-2 d_{5} \delta_{n 3}\right)\right] \\
& +P_{f}\left[\sup _{h \in H_{n, \gamma_{1}}}\left|Z_{n, \gamma_{1}}(h)\right| \geq d_{5} \delta_{n 3} \eta_{n, \gamma_{0}}\left(1-\delta_{n}\right)\right] .
\end{aligned}
$$

For the first term on the right-hand side we apply Lemma 4.3, for

$$
\tau_{n, \gamma_{0}} \leq \eta_{n, \gamma_{0}}\left(1-\delta_{n}\right)\left(1-2 d_{5} \delta_{n 3}\right) \leq c_{1} s_{n, \gamma_{0}} \sqrt{\log n}
$$

(that holds for $c_{1}$ large enough) and by the choice of $\delta_{n 3}$ we get

$$
P_{f}\left[\sup _{h \in H_{n, \gamma_{0}}}\left|Z_{n, \gamma_{0}}(h)\right| \geq \eta_{n, \gamma_{0}}\left(1-\delta_{n}\right)\left(1-2 d_{5} \delta_{n 3}\right)\right] \leq d_{3} \exp \left\{-\frac{q \log n}{4 \gamma_{0}}\left(1-O(1) \delta_{n 3}\right)\right\} \leq d_{3} n^{-\frac{q}{4 \gamma}}
$$

For the second term, $\tau_{n, \gamma_{1}} \leq \sqrt{\frac{q}{2 \gamma_{1}}} s_{n, \gamma_{1}} \sqrt{\log n} \leq d_{5} \delta_{n 3} \eta_{n, \gamma_{0}}\left(1-\delta_{n}\right) \leq c_{2}$ and we apply Lemma 4.5 for $\gamma_{0} \leq \gamma$ as follows

$$
P_{f}\left[\sup _{h \in H_{n, \gamma_{1}}}\left|Z_{n, \gamma_{1}}(h)\right| \geq d_{5} \delta_{n 3} \eta_{n, \gamma_{0}}\left(1-\delta_{n}\right)\right] \leq 8 \exp \left\{-a \frac{\log n}{\gamma}\left(\frac{\delta_{n 3} s_{n, \gamma_{0}}}{s_{n, \gamma_{1}}}\right)^{2}\left(1-\delta_{n}\right)^{2}\right\}
$$

and by the third statement of Lemma 4.7 this exponential is infinitely small with respect to $n^{-\frac{q}{4 \gamma}}$. That allows us to conclude that

$$
p_{1} \leq O(1) n^{-\frac{q}{4 \gamma}}
$$

We finish the proof of the lemma by using Lemma 3.2

$$
p_{2} \leq P_{f}\left[\bar{A}_{n, \gamma_{0}}\right]+P_{f}\left[\bar{A}_{n, \gamma_{1}}\right] \leq 4 \exp \left\{-\frac{n\left((1-\alpha) h_{n} \delta_{n} \rho_{n}\right)^{2}}{2\left\|K_{0}\right\|_{\infty}^{2}}\right\}
$$

which is $o(1) n^{-\frac{q}{4 \gamma}}$ by (3.1). 
Theorem 4.9. We have the following convergence

$$
\sup _{\beta \in B_{N_{n}}} \operatorname{card} B_{-} \sup _{\substack{\gamma \in B_{-} \\ \gamma<\beta}} \frac{\left(1+\delta_{n}\right)^{q} b_{n, \gamma}^{q}+\tau_{n, \gamma}^{q}}{\psi_{n, \beta}^{q}} \sup _{f \in W_{n}(\beta, L)} P_{f}[\hat{\beta}=\gamma] \underset{n \rightarrow \infty}{\rightarrow} 0 .
$$

Proof. Let us use Lemma 4.8 and, respectively, Lemma 3.5, for $\gamma<\beta$

$$
\begin{aligned}
\frac{\left(1+\delta_{n}\right)^{q} b_{n, \gamma}^{q}+\tau_{n, \gamma}^{q}}{\psi_{n, \beta}^{q}} \sup _{f \in W_{n}(\beta, L)} P_{f}[\hat{\beta}=\gamma] & \leq d_{4} \frac{\left(1+\delta_{n}\right)^{q} b_{n, \gamma}^{q}+\tau_{n, \gamma}^{q}}{\psi_{n, \beta}^{q}} \frac{\beta_{N}}{\Delta_{n}} n^{-\frac{q}{4 \gamma}} \\
& \leq O(1)(\log n)^{q / 2} n^{q\left(\frac{1}{4 \gamma}-\frac{1}{4 \beta}\right)} \frac{\beta_{N}}{\Delta_{n}} n^{-\frac{q}{4 \gamma}}
\end{aligned}
$$

We also have $\operatorname{card} B_{-} \leq \frac{\beta_{N}}{\Delta_{n}}$ and then

$$
\operatorname{card} B_{-} \sup _{\substack{\gamma \in B_{-} \\ \gamma<\beta}} \frac{\left(1+\delta_{n}\right)^{q} b_{n, \gamma}^{q}+\tau_{n, \gamma}^{q}}{\psi_{n, \beta}^{q}} \sup _{f \in W_{n}(\beta, L)} P_{f}[\hat{\beta}=\gamma] \leq O(1) \frac{\beta_{N}^{2}(\log n)^{q / 2}}{\Delta_{n}^{2}} n^{-\frac{q}{4 \beta_{N}}}
$$

which is a $o(1)$, by means of relations (2.5) to (2.7). This expression is also independent of $\beta$ in $B$ and then the convergence is uniform.

\section{UPPER BOUND}

Let us prove (2.15) and (2.17) by successive decomposition of the risk and using the tools developed above. Denote $R_{n, \beta}=R_{n, \beta}\left(f_{n}^{*}, \psi_{n, \beta}\right)$. In order to bound from above this expression, we consider the two possibilities when the estimated value $\hat{\beta}$ is greater or less than the true, unknown value of $\beta$. So we write:

$$
R_{n, \beta} \leq R_{n, \beta}^{+}+R_{n, \beta}^{-}
$$

where

$$
\begin{aligned}
& R_{n, \beta}^{+}=\sup _{f \in W_{n}(\beta, L)} \psi_{n, \beta}^{-q} E_{f}\left[\left|f_{n}^{*}\left(x_{0}\right)-f\left(x_{0}\right)\right|^{q} I(\hat{\beta} \geq \beta)\right], \\
& R_{n, \beta}^{-}=\sup _{f \in W_{n}(\beta, L)} \psi_{n, \beta}^{-q} E_{f}\left[\left|f_{n}^{*}\left(x_{0}\right)-f\left(x_{0}\right)\right|^{q} I(\hat{\beta}<\beta)\right] .
\end{aligned}
$$

Thus, the proof of (2.15) consists of the following parts:

$$
\limsup _{n \rightarrow \infty} \sup _{\beta \in B_{-}} R_{n, \beta}^{+} \leq 1
$$

and

$$
\limsup _{n \rightarrow \infty} \sup _{\beta \in B_{-}} R_{n, \beta}^{-}=0 \text {. }
$$




\subsection{Proof of (5.1)}

Under the assumption $\hat{\beta} \geq \beta$, we have $\left|\widehat{f}_{n, \widehat{\beta}}\left(x_{0}\right)-\widehat{f}_{n, \beta}\left(x_{0}\right)\right| \leq \widehat{\eta}_{n, \beta}$, by the definition of the estimator $\hat{\beta}$. Therefore

$$
R_{n, \beta}^{+} \leq \sup _{f \in W_{n}(\beta, L)} \psi_{n, \beta}^{-q} E_{f}\left[\widehat{\eta}_{n, \beta}+\left|\widehat{f}_{n, \beta}\left(x_{0}\right)-f\left(x_{0}\right)\right|\right]^{q}
$$

where $\widehat{f}_{n, \beta}\left(x_{0}\right)$ is the kernel estimator with kernel $K_{\beta}$ and random bandwidth $\widehat{h}_{n, \beta}$.

We shall split again this upper bound, by integrating separately over $A_{n, \beta}$ and $\bar{A}_{n, \beta}$ (the complementary event of $\left.A_{n, \beta}\right)$, respectively. Then

$$
R_{n, \beta}^{+} \leq R_{n, \beta}^{+}\left(A_{n, \beta}\right)+R_{n, \beta}^{+}\left(\bar{A}_{n, \beta}\right)
$$

where

$$
\begin{aligned}
R_{n, \beta}^{+}\left(A_{n, \beta}\right) & =\sup _{f \in W_{n}(\beta, L)} \psi_{n, \beta}^{-q} E_{f}\left[\left(\widehat{\eta}_{n, \beta}+\left|\widehat{f}_{n, \beta}\left(x_{0}\right)-f\left(x_{0}\right)\right|\right)^{q} I\left(A_{n, \beta}\right)\right] \\
R_{n, \beta}^{+}\left(\bar{A}_{n, \beta}\right) & =\sup _{f \in W_{n}(\beta, L)} \psi_{n, \beta}^{-q} E_{f}\left[\left(\widehat{\eta}_{n, \beta}+\left|\widehat{f}_{n, \beta}\left(x_{0}\right)-f\left(x_{0}\right)\right|\right)^{q} I\left(\bar{A}_{n, \beta}\right)\right] .
\end{aligned}
$$

We shall prove that

$$
\limsup _{n \rightarrow \infty} \sup _{\beta \in B_{-}} R_{n, \beta}^{+}\left(A_{n, \beta}\right) \leq 1
$$

and that

$$
\limsup _{n \rightarrow \infty} \sup _{\beta \in B_{-}} R_{n, \beta}^{+}\left(\bar{A}_{n, \beta}\right)=0
$$

Proof of (5.3). We are in the case where the event $A_{n, \beta}$ holds. Then

$$
\begin{aligned}
\widehat{\eta}_{n, \beta}+\left|\widehat{f}_{n, \beta}\left(x_{0}\right)-f\left(x_{0}\right)\right| & \leq \sup _{h \in \mathcal{H}_{n, \beta}}\left[\eta_{n, \beta}\left(\frac{h}{h_{n, \beta}}\right)^{\beta-1 / 2}+\left|f_{n}\left(x_{0}, h, K_{\beta}\right)-f\left(x_{0}\right)\right|\right] \\
& \leq \sup _{h \in \mathcal{H}_{n, \beta}}\left[\eta_{n, \beta}\left(\frac{h}{h_{n, \beta}}\right)^{\beta-1 / 2}+b_{n, \beta}(h)+\left|Z_{n, \beta}(h)\right|\right] \\
& \leq \psi_{n, \beta}\left(1+\delta_{n}\right)+\sup _{h \in H_{n, \beta}}\left|Z_{n, \beta}(h)\right|
\end{aligned}
$$


because $H_{n, \beta} \supseteq \mathcal{H}_{n, \beta}$ by relation (4.1) and $\eta_{n, \beta}+b_{n, \beta}=\psi_{n, \beta}$. Then

$$
\begin{aligned}
R_{n, \beta}^{+}\left(A_{n, \beta}\right) \leq & \sup _{f \in W_{n}(\beta, L)} E_{f}\left[\left(1+\delta_{n}\right)+\psi_{n, \beta}^{-1} \sup _{h \in H_{n, \beta}}\left|Z_{n, \beta}(h)\right|\right]^{q} \\
\leq & \sup _{f \in W_{n}(\beta, L)}\left(1+\delta_{n}+\psi_{n, \beta}^{-1} \tau_{n, \beta}\right)^{q} \\
& +2^{q-1}\left(1+\delta_{n}\right)^{q} \sup _{f \in W_{n}(\beta, L)} P_{f}\left[\sup _{h \in H_{n, \beta}}\left|Z_{n, \beta}(h)\right| \geq \tau_{n, \beta}\right] \\
& +2^{q-1} \sup _{f \in W_{n}(\beta, L)} \psi_{n, \beta}^{-q} E_{f}\left[\left(\sup _{h \in H_{n, \beta}}\left|Z_{n, \beta}(h)\right|\right)^{q} I\left(\sup _{h \in H_{n, \beta}}\left|Z_{n, \beta}(h)\right| \geq \tau_{n, \beta}\right)\right]
\end{aligned}
$$

For the first term on the right-hand side we apply Lemma 3.5 and this term is $1+o(1)$, uniformly in $\beta$ over $B_{-}$.

The last two terms on the right-hand side tend to zero uniformly in $\beta$ over $B_{-}$, as we see from Theorem 4.4 and the particular case of Theorem 4.6. This proves statement (5.3).

Proof of (5.4). We give direct upper bounds by Lemma 3.4 and then apply Lemma 3.2

$$
\begin{aligned}
R_{n, \beta}^{+}\left(\bar{A}_{n, \beta}\right) & \leq \sup _{f \in W_{n}(\beta, L)} \psi_{n, \beta}^{-q}\left(\widehat{\eta}_{n, \beta}+\left|\widehat{f}_{n, \beta}\left(x_{0}\right)-f\left(x_{0}\right)\right|\right)^{q} \sup _{f \in W_{n}(\beta, L)} P_{f}\left[\bar{A}_{n, \beta}\right] \\
& \leq 4 d_{0} n^{d_{1}} \exp \left\{-\frac{n\left(h_{n} \delta_{n} \rho_{n}\right)^{2}}{2\|K\|_{\infty}^{2}}\right\} .
\end{aligned}
$$

This bound is exponentially small uniformly in $\beta$ over $B_{-}$by (3.1), then (5.4) holds.

\subsection{Proof of (5.2)}

As $\{\hat{\beta}<\beta\} \subset \underset{\substack{\gamma \in B_{-} \\ \gamma<\beta}}{\bigcup}\{\hat{\beta}=\gamma\}$, we may write:

$$
\begin{aligned}
R_{n, \beta}^{-} & \leq \sum_{\substack{\gamma \in B_{-} \\
\gamma<\beta}} \sup _{f \in W_{n}(\beta, L)} \psi_{n, \beta}^{-q} E_{f}\left[\left|\widehat{f}_{n, \gamma}\left(x_{0}\right)-f\left(x_{0}\right)\right|^{q} I(\hat{\beta}=\gamma)\right] \\
& \leq \operatorname{card} B_{N_{n}} \sup _{\substack{\gamma \in B_{-} \\
\gamma<\beta}} \sup _{\substack{\gamma \in W_{n}(\beta, L) \\
n, \beta}} \psi_{n, \beta}^{-q} E_{f}\left[\left|\widehat{f}_{n, \gamma}\left(x_{0}\right)-f\left(x_{0}\right)\right|^{q} I(\hat{\beta}=\gamma)\right] \\
& \leq \frac{\beta_{N_{n}}}{\Delta_{n}} \sup _{\substack{\gamma \in B_{-} \\
\gamma<\beta}} R_{n, \beta}^{\gamma},
\end{aligned}
$$

where

$$
R_{n, \beta}^{\gamma}=\sup _{f \in W_{n}(\beta, L)} \psi_{n, \beta}^{-q} E_{f}\left[\left|\widehat{f}_{n, \gamma}\left(x_{0}\right)-f\left(x_{0}\right)\right|^{q} I(\hat{\beta}=\gamma)\right]
$$

Similarly to the proof of (5.3) we integrate over $A_{n, \gamma}$ and $\bar{A}_{n, \gamma}$, respectively. We obtain

$$
R_{n, \beta}^{\gamma} \leq R_{n, \beta}^{\gamma}\left(A_{n, \gamma}\right)+R_{n, \beta}^{\gamma}\left(\bar{A}_{n, \gamma}\right)
$$


with

$$
\begin{aligned}
& R_{n, \beta}^{\gamma}\left(A_{n, \gamma}\right)=\sup _{f \in W_{n}(\beta, L)} \psi_{n, \beta}^{-q} E_{f}\left[\left|\widehat{f}_{n, \gamma}\left(x_{0}\right)-f\left(x_{0}\right)\right|^{q} I(\hat{\beta}=\gamma) I\left(A_{n, \gamma}\right)\right] \\
& R_{n, \beta}^{\gamma}\left(\bar{A}_{n, \gamma}\right)=\sup _{f \in W_{n}(\beta, L)} \psi_{n, \beta}^{-q} E_{f}\left[\left|\widehat{f}_{n, \gamma}\left(x_{0}\right)-f\left(x_{0}\right)\right|^{q} I(\hat{\beta}=\gamma) I\left(\bar{A}_{n, \gamma}\right)\right] .
\end{aligned}
$$

Therefore, $R_{n, \beta}^{-} \leq \frac{\beta_{N_{n}}}{\Delta_{n}} \cdot \sup _{\substack{\gamma \in B_{-} \\ \gamma<\beta}}\left(R_{n, \beta}^{\gamma}\left(A_{n, \gamma}\right)+R_{n, \beta}^{\gamma}\left(\bar{A}_{n, \gamma}\right)\right)$. We shall prove that

$$
\limsup _{n \rightarrow \infty} \sup _{\beta \in B_{-}}\left(\frac{\beta_{N_{n}}}{\Delta_{n}} \sup _{\substack{\gamma \in B_{-} \\ \gamma<\beta}} R_{n, \beta}^{\gamma}\left(A_{n, \gamma}\right)\right)=0
$$

and that

$$
\limsup _{n \rightarrow \infty} \sup _{\beta \in B_{-}}\left(\frac{\beta_{N_{n}}}{\Delta_{n}} \sup _{\substack{\gamma \in B_{-} \\ \gamma<\beta}} R_{n, \beta}^{\gamma}\left(\bar{A}_{n, \gamma}\right)\right)=0 .
$$

Proof of (5.5). The event $A_{n, \gamma}$ is supposed to hold and we write directly that

$$
\left|\widehat{f}_{n, \gamma}\left(x_{0}\right)-f\left(x_{0}\right)\right| \leq \sup _{h \in \mathcal{H}_{n, \gamma}}\left|f_{n}\left(x_{0}, h, K_{\gamma}\right)-f\left(x_{0}\right)\right| \leq \sup _{h \in \mathcal{H}_{n, \gamma}}\left(b_{n, \gamma}(h)+\left|Z_{n, \gamma}(h)\right|\right) .
$$

We use this bound in the expression of $A_{n, \gamma}$ and again relation (4.1)

$$
\begin{aligned}
R_{n, \beta}^{\gamma}\left(A_{n, \gamma}\right) \leq & \sup _{f \in W_{n}(\beta, L)} \psi_{n, \beta}^{-q} E_{f}\left[\left(b_{n, \gamma}\left(1+\delta_{n}\right)+\sup _{h \in H_{n, \gamma}}\left|Z_{n, \gamma}(h)\right|\right)^{q} I(\hat{\beta}=\gamma)\right] \\
\leq & 2^{q-1}\left(1+\delta_{n}\right)^{q} \sup _{f \in W_{n}(\beta, L)}\left(\frac{b_{n, \gamma}^{q}}{\psi_{n, \beta}^{q}} \cdot P_{f}[\hat{\beta}=\gamma]\right) \\
& +2^{q-1} \sup _{f \in W_{n}(\beta, L)} \psi_{n, \beta}^{-q} E_{f}\left[\left(\sup _{h \in H_{n, \gamma}}\left|Z_{n, \gamma}(h)\right|\right)^{q} I(\hat{\beta}=\gamma)\right] \\
\leq & 2^{q-1} \sup _{f \in W_{n}(\beta, L)} \frac{b_{n, \gamma}^{q}\left(1+\delta_{n}\right)^{q}+\tau_{n, \gamma}^{q}}{\psi_{n, \beta}^{q}} \sup _{f \in W_{n}(\beta, L)} P_{f}[\hat{\beta}=\gamma] \\
& +2^{q-1} \sup _{f \in W_{n}(\beta, L)} \psi_{n, \beta}^{-q} E_{f}\left[\left(\sup _{h \in H_{n, \gamma}}\left|Z_{n, \gamma}(h)\right|\right)^{q} I\left(\sup _{h \in H_{n, \gamma}}\left|Z_{n, \gamma}(h)\right| \geq \tau_{n, \gamma}\right)\right] .
\end{aligned}
$$

Then, we have to apply Theorems 4.9 and 4.6. Thus, both terms on the right-hand side tend to 0 , when we multiply by card $B_{n}$ and we take their supremum over $\gamma<\beta$, uniformly over $\beta \in B_{-}$. This finishes the proof of (5.5). 
Proof of (5.6). We apply again Lemma 3.4 together with Lemma 3.2 which allow us to conclude that

$$
\begin{aligned}
R_{n, \beta}^{\gamma}\left(\bar{A}_{n, \gamma}\right) & \leq \sup _{f \in W_{n}(\beta, L)} \psi_{n, \beta}^{-q}\left|\widehat{f}_{n, \gamma}\left(x_{0}\right)-f\left(x_{0}\right)\right|^{q} \sup _{f \in W_{n}(\beta, L)} P_{f}\left[\bar{A}_{n, \gamma}\right] \\
& \leq 4 d_{0} n^{d_{1}} \exp \left\{-\frac{n\left(h_{n} \delta_{n} \rho_{n}\right)^{2}}{2\|K\|_{\infty}^{2}}\right\} .
\end{aligned}
$$

Since those bounds are exponentially small by (3.1) and depend neither on $\gamma$, nor on $\beta$, we have

$$
\sup _{\beta \in B}\left(\frac{\beta_{N_{n}}}{\Delta_{n}} \cdot \sup _{\substack{\gamma \in B \\ \gamma<\beta}} R_{n, \beta}^{\gamma}\left(\bar{A}_{n, \gamma}\right)\right)=o(1) .
$$

\subsection{Proof of (2.17)}

For the case $\beta=\beta_{N_{n}}=\beta_{N}$, we have very similarly to (5.1):

$$
\begin{aligned}
R_{n, \beta_{N}}^{+}\left(f_{n}^{*}, \psi_{n, \beta_{N}}\right) & =\sup _{f \in W_{n}(\beta, L)} \psi_{n, \beta_{N}}^{-q} E_{f}\left[\left|f_{n}^{*}\left(x_{0}\right)-f\left(x_{0}\right)\right|^{q} I\left(\widehat{\beta}=\beta_{N}\right)\right] \\
& =\sup _{f \in W_{n}(\beta, L)} \psi_{n, \beta_{N}}^{-q} E_{f}\left[\left|f_{n, \beta_{N}}\left(x_{0}\right)-f\left(x_{0}\right)\right|^{q}\right] \\
& \leq \sup _{f \in W_{n}(\beta, L)} \frac{b_{n, \beta_{N}}^{q}+c(q) s_{n, \beta_{N}}^{q}}{\psi_{n, \beta_{N}}^{q}}<\infty,
\end{aligned}
$$

by Lemma 3.3. Similarly to (5.2),

$$
R_{n, \beta_{N}}^{-}\left(f_{n}, \psi_{n, \beta_{N}}\right)=\sup _{f \in W_{n}(\beta, L)} \psi_{n, \beta_{N}}^{-q} E_{f}\left[\left|f_{n}^{*}\left(x_{0}\right)-f\left(x_{0}\right)\right|^{q} I\left(\widehat{\beta}<\beta_{N}\right)\right] \leq \frac{\beta_{N}}{\Delta_{n}} \sup _{\gamma \in B_{-}} R_{n, \beta_{N}}^{\gamma} \leq o(1) .
$$

Indeed, the proof goes exactly like in the case $\beta \in B_{-}$, for $\beta=\beta_{N}$ and $\gamma \in B_{-}$.

The proof of the upper bounds is now completed.

\section{LOWER BOUND}

The proof of the inequality (2.16) will now be given. In order to bound from below the minimax risk $R_{n, \beta}\left(f_{n}, \psi_{n, \beta}\right)$ in $(2.2)$, it suffices to bound it over a suitably chosen subset of probability densities in $W_{n}(\beta, L)$.

\subsection{Introduction}

Let $f$ be a positive probability density on $\mathbb{R}$, infinitely continuously differentiable. For $\beta$ in $B_{N_{n}}$, we denote $\left\|f^{(\beta)}\right\|_{2}^{2}=\frac{1}{2 \pi} \int_{\mathbb{R}}(\mathcal{F}(f)(x))^{2}|x|^{2 \beta} \mathrm{d} x$. Let us consider the extreme values: $\beta_{1}$ and $\beta_{N-1}=\beta_{N_{n}-1}$ (the last element of the set $\left.B_{-}\right)$and let $f$ be such that both $\left\|f^{\left(\beta_{1}\right)}\right\|_{2} \leq L$ and $\left\|f^{\left(\beta_{N-1}\right)}\right\|_{2} \leq L$ hold, for each $n$. Let $\delta \in(0,1 / 2)$ be a small constant and define

$$
f_{n, 0}(x)=\left(\frac{\delta}{2}\right)^{\frac{1}{\beta_{1}+1 / 2}} f\left(x\left(\frac{\delta}{2}\right)^{\frac{1}{\beta_{1}+1 / 2}}\right),
$$

such that $f_{n, 0}\left(x_{0}\right) \geq \rho_{n}$. 
Lemma 6.1. Define $K_{\beta}^{*}(x)=\nu_{\beta}^{-1}(2 \beta-1)^{\frac{1}{4 \beta}} K_{\beta}\left((2 \beta-1)^{\frac{1}{2 \beta}} x\right)$, where $K_{\beta}$ is given by (2.12). Then $\left\|K_{\beta}^{*}\right\|_{2}$ $=1,\left\|K_{\beta}^{*(\beta)}\right\|_{2}=1$ and

$$
K_{\beta}^{*}(0)=b_{\beta}(2 \beta)(2 \beta-1)^{-\frac{2 \beta-1}{4 \beta}} .
$$

Indeed, it suffices to recall that $\left\|K_{\beta}\right\|_{2}=\nu_{\beta}$ and that $\nu_{\beta}^{2}=(2 \beta-1) b_{\beta}^{2}$. We can modify the function $K_{\beta}^{*}$ in order to get the compact supported function $\widetilde{K}_{\beta}$ having the following properties.

Lemma 6.2. (Tsybakov [30]) For any fixed $\delta \in\left(0, \frac{1}{2}\right)$ and $\beta>\frac{1}{2}$ there exists a number $D=D(\beta, \delta)>0$ and an integrable function $\widetilde{K}_{\beta}$ supported on $(-D, D)$ such that $\left\|\widetilde{K}_{\beta}\right\|_{2} \leq 1-\delta / 2,\left\|\widetilde{K}_{\beta}^{(\beta)}\right\|_{2} \leq 1-\delta / 2$ and $(1-\delta) K_{\beta}^{*}(0) \leq \widetilde{K}_{\beta}(0) \leq K_{\beta}^{*}(0)$.

By this lemma, we get an integrable kernel, $\widetilde{K}_{\beta_{1}} \in W\left(\beta_{1}, 1\right)$, satisfying the same conditions. Let

$$
g_{n, \beta_{1}}(x)=L \widetilde{h}_{n, \beta_{1}}^{\beta_{1}-\frac{1}{2}} \cdot \widetilde{K}_{\beta_{1}}\left(\frac{x-x_{0}}{\widetilde{h}_{n, \beta_{1}}}\right),
$$

where $\widetilde{h}_{n, \beta_{1}}=\widetilde{k}_{\beta_{1}}\left(\frac{f_{n, 0}\left(x_{0}\right) \cdot \log n}{n}\right)^{\frac{1}{2 \beta_{1}}}$ and $\widetilde{k}_{\beta_{1}}=\left(\frac{q}{L^{2} \cdot 2 \beta_{1}}\right)^{\frac{1}{2 \beta_{1}}}$.

Denote $\varepsilon_{n, \beta_{1}}=\int_{\mathbb{R}} g_{n, \beta_{1}}(x) \mathrm{d} x$ which is finite by the previous lemma. Consider

$$
f_{n, 1}(x)=f_{n, 0}(x)\left(1-\varepsilon_{n, \beta_{1}}\right)+g_{n, \beta_{1}}(x) .
$$

Lemma 6.3. Let $f_{n, 0}, g_{n, \beta_{1}}$ and $\varepsilon_{n, \beta_{1}}$ be defined as above. Then, $f_{n, 0} \in W_{n}\left(\beta_{N-1}, L\right)$ and $f_{n, 1} \in W_{n}\left(\beta_{1}, L\right)$, $\varepsilon_{n, \beta_{1}}=O\left(\widetilde{h}_{n, \beta_{1}}^{\beta_{1}+\frac{1}{2}}\right)$ and $(1-\delta) \psi_{n, \beta_{1}} \leq g_{n, \beta_{1}}\left(x_{0}\right) \leq \psi_{n, \beta_{1}}$.

Proof. We can easily prove that $f_{n, 0}$ is also a positive density function, infinitely continuously differentiable on $\mathbb{R}$. Moreover,

$$
\left\|f_{n, 0}^{\left(\beta_{1}\right)}\right\|_{2}=\frac{\delta}{2}\left\|f^{\left(\beta_{1}\right)}\right\|_{2} \leq \frac{L \delta}{2}
$$

Consequently, $f_{n, 0} \in W_{n}\left(\beta_{1}, L \frac{\delta}{2}\right)$ and obviously $f_{n, 0} \in W_{n}\left(\beta_{N-1}, L\right)$, too.

We can see that

$$
\varepsilon_{n, \beta_{1}}=L \widetilde{h}_{n, \beta_{1}}^{\beta_{1}+\frac{1}{2}} \int_{\mathbb{R}} \widetilde{K}_{\beta_{1}}(x) \mathrm{d} x=O\left(\widetilde{h}_{n, \beta_{1}}^{\beta_{1}+\frac{1}{2}}\right)
$$

and that $g_{n, \beta_{1}}\left(x_{0}\right)=L \widetilde{h}_{n, \beta_{1}}^{\beta_{1}-\frac{1}{2}} \widetilde{K}_{\beta_{1}}(0)$. Remark that $\psi_{n, \beta_{1}}=L \widetilde{h}_{n, \beta_{1}}^{\beta_{1}-\frac{1}{2}} K_{\beta_{1}}(0)$ and by Lemma 6.3

$$
(1-\delta) \psi_{n, \beta_{1}} \leq g_{n, \beta_{1}}\left(x_{0}\right) \leq \psi_{n, \beta_{1}} .
$$

Remark also that $\left\|g_{n, \beta_{1}}^{\left(\beta_{1}\right)}\right\|_{2}=L\left\|\widetilde{K}_{\beta_{1}}^{\left(\beta_{1}\right)}\right\|_{2} \leq L\left(1-\frac{\delta}{2}\right)$.

It is easy to prove that $f_{n, 1}$ is a density function, positive for $n$ large enough and that

$$
f_{n, 1}\left(x_{0}\right) \geq \rho_{n}\left(1-\varepsilon_{n, \beta_{1}}\right)+g_{n, \beta_{1}}\left(x_{0}\right) \geq \rho_{n}
$$


since $g_{n, \beta_{1}}\left(x_{0}\right) \geq \rho_{n} \varepsilon_{n, \beta_{1}}$, for $n$ large enough. Moreover,

$$
\left\|f_{n, 1}^{\left(\beta_{1}\right)}\right\|_{2} \leq\left(1-\varepsilon_{n, \beta_{1}}\right)\left\|f_{n, 0}^{\left(\beta_{1}\right)}\right\|_{2}+\left\|g_{n, \beta_{1}}^{\left(\beta_{1}\right)}\right\|_{2} \leq L \frac{\delta}{2}+L\left(1-\frac{\delta}{2}\right)=L
$$

Then $f_{n, 1} \in W_{n}\left(\beta_{1}, L\right)$.

\subsection{Proof of the lower bound}

For chosen $f_{n, 0} \in W_{n}\left(\beta_{N-1}, L\right)$ and $f_{n, 1} \in W_{n}\left(\beta_{1}, L\right)$ as in the previous section, we have

$$
\begin{aligned}
\inf _{\widehat{f}_{n}} \sup _{\beta \in B} R_{n, \beta}\left(\widehat{f}_{n}, \psi_{n, \beta}\right) & \geq \inf _{\widehat{f}_{n}} \max _{\beta \in\left\{\beta_{1}, \beta_{N-1}\right\}} R_{n, \beta}\left(\widehat{f}_{n}, \psi_{n, \beta}\right) \\
& \geq \inf _{\widehat{f}_{n}} \max \left\{E_{f_{n, 0}}\left[\psi_{n, \beta_{N-1}}^{-q} \cdot\left|\widehat{f}_{n}\left(x_{0}\right)-f_{n, 0}\left(x_{0}\right)\right|^{q}\right],\right. \\
& \left.E_{f_{n, 1}}\left[\psi_{n, \beta_{1}}^{-q} \cdot\left|\widehat{f}_{n}\left(x_{0}\right)-f_{n, 1}\left(x_{0}\right)\right|^{q}\right]\right\} .
\end{aligned}
$$

Let us denote:

$$
T_{n}=\psi_{n, \beta_{1}}^{-1} \cdot\left|\widehat{f}_{n}\left(x_{0}\right)-f_{n, 0}\left(x_{0}\right)\right| \text { and } q_{n}=\frac{\psi_{n, \beta_{1}}}{\psi_{n, \beta_{N-1}}}
$$

Remark that $q_{n} \geq a\left(\frac{n}{\log n}\right)^{\frac{\beta_{N-1}-\beta_{1}}{4 \beta_{1} \beta_{N-1}}} \rightarrow+\infty$, when $n \rightarrow \infty$. Then, we may write

$$
\begin{aligned}
\inf _{\widehat{f}_{n}} \sup _{\beta \in B} R_{n, \beta}\left(\widehat{f}_{n}, \psi_{n, \beta}\right) & \geq \inf _{T_{n}} \max \left\{E_{0}\left|q_{n} T_{n}\right|^{q}, E_{1}\left|T_{n}-\frac{f_{n, 1}\left(x_{0}\right)-f_{n, 0}\left(x_{0}\right)}{\psi_{n, \beta_{1}}}\right|^{q}\right\} \\
& \geq \inf _{T_{n}} \max \left\{E_{0}\left|q_{n} T_{n}\right|^{q}, E_{1}\left|T_{n}-\theta_{1}\right|^{q}\right\}
\end{aligned}
$$

with the notation $E_{0}=E_{f_{n, 0}}, E_{1}=E_{f_{n, 1}}$ (with the associated probability laws $P_{0}$ and $P_{1}$ ) and $\theta_{1}=$ $\left(g_{n, \beta_{1}}\left(x_{0}\right)-\varepsilon_{n, \beta_{1}} \cdot f_{n, 0}\left(x_{0}\right)\right) / \psi_{n, \beta_{1}}$. Let us denote $R_{n}\left(T_{n}, \theta_{1}\right)$ the right-hand side term in $(6.1)$, where inf denotes the infimum over all random functions $T_{n}$.

Following the proof in Theorem 6, Tsybakov [30] we state here a similar result.

Lemma 6.4. Let the numbers $q_{n}, q>0, \tau>0,0<\delta<\frac{1}{2}$ be fixed, let $\theta_{1}$ be a real number such that $\left|\theta_{1}\right| \geq 1-2 \delta$ and let $P_{0}, P_{1}$ be such that $P_{1}\left[\frac{\mathrm{d} P_{0}}{\mathrm{~d} P_{1}} \geq \tau\right] \geq 1-\delta$. Then

$$
R_{n}\left(T_{n}, \theta_{1}\right) \geq \frac{(1-\delta) \tau q_{n}^{q}(2 \delta)^{q}(1-2 \delta)^{2 q}}{(1-2 \delta)^{q}+\tau q_{n}^{q}(2 \delta)^{q}}
$$

Proof. Remark that

$$
\left|\theta_{1}\right|=\left|\frac{g_{n, \beta_{1}}\left(x_{0}\right)}{\psi_{n, \beta_{1}}}-\frac{\varepsilon_{n, \beta_{1}} \cdot f_{n, 0}\left(x_{0}\right)}{\psi_{n, \beta_{1}}}\right| \geq|| \frac{g_{n, \beta_{1}}\left(x_{0}\right)}{\psi_{n, \beta_{1}}}|-| \frac{\varepsilon_{n, \beta_{1}} \cdot f_{n, 0}\left(x_{0}\right)}{\psi_{n, \beta_{1}}}|| .
$$

By Lemma 6.3, $1-\delta<\left|\frac{g_{n, \beta_{1}}\left(x_{0}\right)}{\psi_{n, \beta_{1}}}\right|<1$. By the definition of $\varepsilon_{n, \beta_{1}}, \frac{\varepsilon_{n, \beta_{1}} \cdot f_{n, 0}\left(x_{0}\right)}{\psi_{n, \beta_{1}}} \leq O\left(\widetilde{h}_{n, \beta_{1}}\right)=o(1)$ which allows us to consider $\left|\frac{\varepsilon_{n, \beta_{1}} \cdot f_{n, 0}\left(x_{0}\right)}{\psi_{n, \beta_{1}}}\right|<\delta$, for sufficiently large $n$. Then $\left|\theta_{1}\right| \geq 1-2 \delta$. 
Let $B=\left\{\left|T_{n}\right| \geq 2 \delta(1-2 \delta)\right\}$. For $\delta<\frac{1}{2}$, the event $\left\{\left|T_{n}-\theta_{1}\right| \geq(1-2 \delta)^{2}\right\}$ contains $\bar{B}$ and we deduce that

$$
P_{1}\left[\left|T_{n}-\theta_{1}\right| \geq(1-2 \delta)^{2}\right] \geq P_{1}[\bar{B}] \text {. }
$$

Then:

$$
R_{n}\left(T_{n}, \theta_{1}\right) \geq \inf _{T_{n}} \max \left\{q_{n}^{q}(2 \delta)^{q}(1-2 \delta)^{q} \cdot P_{0}[B],(1-2 \delta)^{2 q} \cdot P_{1}[B]\right\} .
$$

Moreover, let $A=\left\{\frac{d P_{0}}{d P_{1}} \geq \tau\right\}$ and suppose for the moment that

$$
P_{1}[A] \geq 1-\delta
$$

for arbitrary small $\delta \in\left(0, \frac{1}{2}\right)$, and $\tau>0$. Then

$$
P_{0}[B]=E_{1}\left[\frac{\mathrm{d} P_{0}}{\mathrm{~d} P_{1}} I(B)\right] \geq \tau P_{1}[A \cap B] \geq \tau\left(P_{1}[B]-\delta\right)
$$

and we use this to bound from below the right hand expression in (6.3) to get

$$
\begin{aligned}
R_{n}\left(T_{n}, \theta_{1}\right) & \geq \inf _{T_{n}} \max \left\{\left(q_{n} 2 \delta(1-\delta)\right)^{q} \tau\left(P_{1}[B]-\delta\right),(1-2 \delta)^{2 q} P_{1}[\bar{B}]\right\} \\
& \geq \inf _{0 \leq t \leq 1} \max \left\{\left(q_{n} 2 \delta(1-\delta)\right)^{q} \tau(t-\delta),(1-2 \delta)^{2 q}(1-t)\right\} \\
& \geq \frac{(1-\delta) \tau q_{n}^{q}(2 \delta)^{q}(1-2 \delta)^{2 q}}{(1-2 \delta)^{q}+\tau q_{n}^{q}(2 \delta)^{q}} .
\end{aligned}
$$

Let us prove that (6.4) holds for $n$ large enough, $\tau>0$. We write

$$
P_{1}[A]=P_{1}\left[\prod_{i=1}^{n} \frac{f_{n, 0}\left(X_{i}\right)}{f_{n, 1}\left(X_{i}\right)} \geq \tau\right]=P_{1}\left[\sum_{i=1}^{n} \frac{1}{\sqrt{\log n}} \log \frac{f_{n, 0}\left(X_{i}\right)}{f_{n, 1}\left(X_{i}\right)} \geq \frac{\log \tau}{\sqrt{\log n}}\right] .
$$

Consider the random variables $Z_{n, i}=\frac{1}{\sqrt{\log n}} \log \frac{f_{n, 0}}{f_{n, 1}}\left(X_{i}\right)$, for $i=1, \ldots, n, n \geq 1$, which are independent and identically distributed variables within each series. Let $V_{1}\left[Z_{n, i}\right]$ be the variance with respect to the distribution of $X_{1}, \ldots, X_{n}$ when the underlying probability density is $f_{n, 1}$ and let $U_{n, i}=\left(Z_{n, i}-E_{1}\left[Z_{n, i}\right]\right) / \sigma_{n}$.

Lemma 6.5. We have, for arbitrary small $\delta \in\left(0, \frac{1}{2}\right)$ :
1. $\sum_{i=1}^{n} E_{1}\left[Z_{n, i}\right] \geq-\sqrt{\log n} \frac{q}{4 \beta_{1}}\left(1-\frac{\delta^{2}}{4}\right)^{2}$;
2. $\sigma_{n}^{2}=\sum_{i=1}^{n} V_{1}\left[Z_{n, i}\right] \leq \frac{q}{2 \beta_{1}}\left(1-\frac{\delta^{2}}{4}\right)^{2}$;
3. $E_{1}\left[\left|U_{n, i}\right|^{3}\right]<+\infty$ and $\lim _{n \rightarrow \infty} \sum_{i=1}^{n} E_{1}\left[\left|U_{n, i}\right|^{3}\right]=0$.

Proof. 1. Let us recall that there exists a fixed $a_{0}(\delta) \in(0,1)$ such that

$$
-x-\frac{x^{2}}{2}\left(1+\frac{\delta}{2}\right) \leq \log (1-x) \leq-x
$$


for any $0<x<a_{0}(\delta)$. Then

$$
\sum_{i=1}^{n} E_{1}\left[Z_{n, i}\right]=\frac{n}{\sqrt{\log n}}\left[-\log \left(1-\varepsilon_{n, \beta_{1}}\right)+\int \log \left(1-\frac{g_{n, \beta_{1}}}{f_{n, 1}}(x)\right) f_{n, 1}(x) \mathrm{d} x\right]
$$

As $\varepsilon_{n, \beta_{1}} \rightarrow 0$ and $\sup _{x} g_{n, \beta_{1}} / f_{n, 1}(x) \rightarrow 0$ when $n \rightarrow \infty$, we may suppose without loss of generality that they are smaller than $a_{0}(\delta)$ and apply the inequality (6.5). Then, for $n$ large enough,

$$
\begin{aligned}
\sum_{i=1}^{n} E_{1}\left[Z_{n, i}\right] & \geq-\frac{1}{2}\left(1+\frac{\delta}{2}\right) \frac{n}{\sqrt{\log n}} \int \frac{g_{n, \beta_{1}}^{2}}{f_{n, 1}}(x) \mathrm{d} x \\
& \geq-\frac{1}{2}\left(1+\frac{\delta}{2}\right) \frac{q f_{n, 0}\left(x_{0}\right)}{2 \beta_{1}} \frac{\sqrt{\log n}}{\widetilde{h}_{n, \beta_{1}}} \int \widetilde{K}_{\beta_{1}}^{2}\left(\frac{x-x_{0}}{\widetilde{h}_{n, \beta_{1}}}\right) \frac{\mathrm{d} x}{f_{n, 1}(x)} \\
& \geq-\sqrt{\log n}\left(1+\frac{\delta}{2}\right)^{2} \frac{q}{4 \beta_{1}}\left\|\widetilde{K}_{\beta_{1}}\right\|_{2}^{2}(1+o(1)) \\
& \geq-\sqrt{\log n}\left(1-\frac{\delta^{2}}{4}\right)^{2} \frac{q}{4 \beta_{1}}(1+o(1)),
\end{aligned}
$$

where we used Lemma 6.2, Bochner's lemma and the fact that $f_{n, 0} / f_{n, 1}\left(x_{0}\right)=o(1)$ and becomes less than $1+\delta / 2$ when $n$ is large enough.

2 . For the variance, we have similarly

$$
\begin{aligned}
\sigma_{n}^{2} & \leq \frac{n}{\log n} E_{1}\left[\log ^{2}\left(1-\frac{g_{n, \beta_{1}}}{f_{n, 1}}\left(X_{1}\right)\right)\right] \leq \frac{n}{\log n}\left(\int \frac{g_{n, \beta_{1}}^{2}}{f_{n, 1}}(x) \mathrm{d} x+\frac{1}{2}\left(1+\frac{\delta}{2}\right) \int \frac{g_{n, \beta_{1}}^{4}}{f_{n, 1}}(x) \mathrm{d} x\right) \\
& \leq\left(1+\frac{\delta}{2}\right)^{2} \frac{q}{2 \beta_{1}}\left\|\widetilde{K}_{\beta_{1}}\right\|_{2}^{2} \leq\left(1-\frac{\delta^{2}}{4}\right)^{2} \frac{q}{2 \beta_{1}}
\end{aligned}
$$

for $n$ large enough.

3. By similar considerations, we get:

$$
E_{1}\left[\log ^{2}\left(1-\frac{g_{n, \beta_{1}}}{f_{n, 1}}\left(X_{1}\right)\right)\right] \geq \int \frac{g_{n, \beta_{1}}^{2}}{f_{n, 1}}(x) \mathrm{d} x \geq \frac{\log n}{n}\left(1-\frac{\delta}{2}\right) \frac{q}{2 \beta_{1}}\left\|\widetilde{K}_{\beta_{1}}\right\|_{2}^{2}
$$

and

$$
\left[E_{1} \log \left(1-\frac{g_{n, \beta_{1}}}{f_{n, 1}}\left(X_{1}\right)\right)\right]^{2} \leq\left(-\varepsilon_{n, \beta_{1}}-\frac{1}{2}\left(1+\frac{\delta}{2}\right) \int \frac{g_{n, \beta_{1}}^{2}}{f_{n, 1}}(x) \mathrm{d} x\right)^{2}=o(1) \frac{\log n}{n}
$$

From those two last inequalities, we conclude that $\sigma_{n}^{2} \geq\left(1-\frac{\delta}{2}\right)^{2} \frac{q}{2 \beta_{1}}\left\|\widetilde{K}_{\beta_{1}}\right\|_{2}^{2}$ for $n$ large enough. Then there exists $\liminf _{n \rightarrow \infty} \sum_{i=1}^{n} V_{1}\left[Z_{n, i}\right]=\sigma^{2}>0$, for $\delta \in(0,1 / 2)$.Then

$$
E_{1}\left[\left|U_{i, n}\right|^{3}\right] \leq \frac{O(1)}{(\log n)^{3 / 2}} E_{1}\left|\log \left(1-\frac{g_{n, \beta_{1}}}{f_{n, 1}}\left(X_{i}\right)\right)\right|^{3}
$$


Let us note that

$$
\begin{aligned}
E_{1}\left[\left|\log \left(1-\frac{g_{n, \beta_{1}}}{f_{n, 1}}\left(X_{i}\right)\right)\right|^{3}\right] & \leq O(1) \int \frac{\left|g_{n, \beta_{1}}\right|^{3}}{f_{n, 1}^{2}}(x) \mathrm{d} x \\
& \leq O(1) \widetilde{h}_{n, \beta_{1}}^{3 \beta_{1}-1 / 2} \frac{1}{\widetilde{h}_{n, \beta_{1}}} \int\left|\widetilde{K}_{\beta_{1}}\left(\frac{x-x_{0}}{\widetilde{h}_{n, \beta_{1}}}\right)\right|^{3} \frac{\mathrm{d} x}{f_{n, 1}^{2}(x)} \\
& \leq O(1)\left(\frac{\log n}{n}\right)^{\frac{3 \beta_{1}-1 / 2}{2 \beta_{1}}}=o(1) .
\end{aligned}
$$

Thus

$$
\sum_{i=1}^{n} E_{1}\left[\left|U_{i, n}\right|^{3}\right] \leq O(1)\left(\frac{1}{\log n}\right)^{\frac{1}{4 \beta_{1}}}\left(\frac{1}{n}\right)^{\frac{\beta_{1}-1 / 2}{2 \beta_{1}}}=o(1)
$$

So, the variables $\left(U_{n, i}\right)_{1 \leq i \leq n, n \geq 1}$ are centered, $\sum_{i=1}^{n} V_{1}\left[U_{n, i}\right]=1$ and they satisfy the property 3 of Lemma 6.5 . Then we may apply Lyapounov's central limit theorem and get the following convergence in law to the standard Gaussian distribution:

$$
U_{n}=\sum_{i=1}^{n} U_{n, i} \stackrel{D}{\rightarrow} N(0,1), \text { as } n \rightarrow \infty
$$

We take $m_{n}=\frac{\log \tau-n \cdot E_{1} Z_{n, 1}}{\sqrt{n \cdot V_{1} Z_{n, 1}}}$ and see that

$$
P_{1}[A]=P_{1}\left[U_{n} \geq \frac{\log \tau-n E_{1} Z_{n, 1}}{\sqrt{n V_{1} Z_{n, 1}}}\right]=P_{1}\left[U_{n} \geq m_{n}\right]
$$

Let us choose $\tau=n^{-\xi}, \xi=-\frac{q}{4 \beta_{1}}\left(1-\frac{\delta^{2}}{4}\right)$ and use Lemma 6.5

$$
m_{n} \leq-\sqrt{\log n} \frac{1}{2} \sqrt{\frac{q}{2 \beta_{1}}} \frac{\delta^{2}}{4} .
$$

Then, it is easy to see that $m_{n} \rightarrow-\infty$, for arbitrarily small $\delta \in(0,1 / 2)$ which implies that $P_{1}[A] \underset{n \rightarrow \infty}{\rightarrow} 1$. This proves that (6.4) holds for $n$ large enough and $\delta \in(0,1 / 2)$ arbitrarily small.

By (6.1) and (6.2), we conclude that:

$$
\inf _{\widehat{f}_{n}} \sup _{\beta \in B} R_{n, \beta}\left(\widehat{f}_{n}, \psi_{n, \beta}\right) \geq \frac{(1-\delta) \tau q_{n}^{q}(2 \delta)^{q}(1-2 \delta)^{2 q}}{(1-2 \delta)^{q}+\tau q_{n}^{q}(2 \delta)^{q}} .
$$

As, furthermore:

$$
\begin{aligned}
\liminf _{n \rightarrow \infty} \tau q_{n}^{q} & \geq \liminf _{n \rightarrow \infty} n^{-\frac{q}{4 \beta_{1}}\left(1-\frac{\delta^{2}}{4}\right)}\left(\frac{\psi_{n, \beta_{1}}}{\psi_{n, \beta_{N-1}}}\right)^{q} \\
& \geq \liminf _{n \rightarrow \infty} \exp \left[\frac{q}{4} \log n\left(\frac{1}{\beta_{1}} \frac{\delta^{2}}{4}-\frac{1}{\beta_{N-1}}-\frac{\log \log n}{\log n}\left(\frac{1}{\beta_{1}}-\frac{1}{\beta_{N-1}}\right)\right)\right]
\end{aligned}
$$


which is $\infty$, for arbitrarily small $\delta \in(0,1 / 2)$. Then by (6.6) we get the stated result (2.16).

The author is very grateful to Alexandre Tsybakov for his generous help.

\section{REFERENCES}

[1] A. Barron, L. Birge and P. Massart, Risk bounds for model selection via penalization. Probab. Theory Related Fields 113 (1995) 301-413.

[2] O.V. Besov, V.L. Il'in and S.M. Nikol'skii, Integral representations of functions and imbedding theorems. J. Wiley, New York (1978).

[3] L. Birge and P. Massart, From model selection to adaptive estimation, Festschrift fur Lucien Le Cam. Springer (1997) 55-87.

[4] L.D. Brown and M.G. Low, A constrained risk inequality with application to nonparametric functional estimation. Ann. Statist. 24 (1996) 2524-2535.

[5] C. Butucea, The adaptive rates of convergence in a problem of pointwise density estimation. Statist. Probab. Lett. 47 (2000) 85-90.

[6] C. Butucea, Numerical results concerning a sharp adaptive density estimator. Comput. Statist. 1 (2001).

[7] L. Devroye and G. Lugosi, A universally acceptable smoothing factor for kernel density estimates. Ann. Statist. 24 (1996) 2499-2512.

[8] D.L. Donoho, I. Johnstone, G. Kerkyacharian and D. Picard, Wavelet shrinkage: Asymptopia? J. R. Stat. Soc. Ser. B Stat. Methodol. 57 (1995) 301-369.

[9] D.L. Donoho, I. Johnstone, G. Kerkyacharian and D. Picard, Density estimation by wavelet thresholding. Ann. Statist. 24 (1996) 508-539.

[10] D.L. Donoho and M.G. Low, Renormalization exponents and optimal pointwise rates of convergence. Ann. Statist. 20 (1992) 944-970.

[11] S.Yu. Efromovich, Nonparametric estimation of a density with unknown smoothness. Theory Probab. Appl. 30 (1985) $557-568$.

[12] S.Yu. Efromovich and M.S. Pinsker, An adaptive algorithm of nonparametric filtering. Automat. Remote Control 11 (1984) 1434-1440.

[13] A. Goldenshluger and A. Nemirovski, On spatially adaptive estimation of nonparametric regression. Math. Methods Statist. 6 (1997) $135-170$.

[14] G.K. Golubev, Adaptive asymptotically minimax estimates of smooth signals. Problems Inform. Transmission 23 (1987) 57-67.

[15] G.K. Golubev, Quasilinear estimates for signals in $\mathbb{L}_{2}$. Problems Inform. Transmission 26 (1990) 15-20.

[16] G.K. Golubev, Nonparametric estimation of smooth probability densities in $\mathbb{L}_{2}$. Problems Inform. Transmission 28 (1992) 44-54.

[17] G.K. Golubev and M. Nussbaum, Adaptive spline estimates in a nonparametric regression model. Theory Probab. Appl. 37 (1992) 521-529.

[18] I.A. Ibragimov and R.Z. Hasminskii, Statistical estimation: Asymptotic theory. Springer-Verlag, New York (1981).

[19] A. Juditsky, Wavelet estimators: Adapting to unknown smoothness. Math. Methods Statist. 6 (1997) 1-25.

[20] G. Kerkyacharian and D. Picard, Density estimation by kernel and wavelet method, optimality in Besov space. Statist. Probab. Lett. 18 (1993) 327-336.

[21] G. Kerkyacharian, D. Picard and K. Tribouley, $\mathbb{L}_{p}$ adaptive density estimation. Bernoulli 2 (1996) 229-247.

[22] J. Klemelä and A.B. Tsybakov, Sharp adaptive estimation of linear functionals, Prépublication 540. LPMA Paris 6 (1999).

[23] O.V. Lepskii, On a problem of adaptive estimation in Gaussian white noise. Theory Probab. Appl. 35 (1990) 454-466.

[24] O.V. Lepskii, Asymptotically minimax adaptive estimation I: Upper bounds. Optimally adaptive estimates. Theory Probab. Appl. 36 (1991) 682-697.

[25] O.V. Lepskii, On problems of adaptive estimation in white Gaussian noise. Advances in Soviet Math. Amer. Math. Soc. 12 (1992b) 87-106.

[26] O.V. Lepski and B.Y. Levit, Adaptive minimax estimation of infinitely differentiable functions. Math. Methods Statist. 7 (1998) 123-156.

[27] O.V. Lepski, E. Mammen and V.G. Spokoiny, Optimal spatial adaptation to inhomogeneous smoothness: An approach based on kernel estimates with variable bandwidth selectors. Ann. Statist. 25 (1997) 929-947.

[28] O.V. Lepski and V.G. Spokoiny, Optimal pointwise adaptive methods in nonparametric estimation. Ann. Statist. 25 (1997) 2512-2546.

[29] D. Pollard, Convergence of Stochastic Processes. Springer-Verlag, New York (1984).

[30] A.B. Tsybakov, Pointwise and sup-norm sharp adaptive estimation of functions on the Sobolev classes. Ann. Statist. 26 (1998) 2420-2469.

[31] S. Van de Geer, A maximal inequality for empirical processes, Technical Report TW 9505. University of Leiden, Leiden (1995). 